\title{
Experimental and numerical investigation on the thermal conduction properties of 2.5D angle-interlock woven composites
}

\author{
Kai Dong ${ }^{1}$, Kui Liu ${ }^{1,2},{\text { Lijian } \operatorname{Pan}^{1} \text {, Bohong Gu }}^{1}$, Baozhong Sun ${ }^{1}$ * \\ ${ }^{1}$ State Key Laboratory for Modification of Chemical Fibers and Polymer Materials, College of \\ Textiles, Donghua University, Shanghai, China, 201620 \\ ${ }^{2}$ Shanghai Aircraft Manufacturing Co., Ltd, Shanghai 200436, China

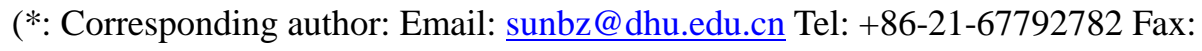 \\ +86-21-67792627)
}

\begin{abstract}
The thermal conduction properties of 2.5D angle-interlock woven composites (2.5DAWC) were investigated along warp, weft and thickness directions both from experimental measurement and finite element analyses (FEA). A self-designed apparatus was established to measure the thermal conductivity of 2.5DAWC. The multi-scale FEA models were selected from representative volume elements (RVE) of matrix, yarns and composites by detailed geometrical structural analyses. The micro-scale models including the matrix-voids RVE and the fiber-matrix RVE were used to calculate the thermal properties of resin matrix and yarns, respectively. The meso-scale model was created to analyze the overall thermal conduction behaviors of 2.5DAWC, including the temperature and heat flux distributions. The effects of voids and interface thermal contact resistance on the thermal conductivity were also considered and analyzed in this paper. The results from FEA showed reasonable agreement with the experimental with an error of less than 5\%. The methodology of this paper could be applied to understand the thermal conduction behaviors of complicated structural composites, and also can be used to predict the coupled thermal-mechanical properties of composites.
\end{abstract}

Keywords: Polymer-matrix composites (PMCs); Thermal properties; Finite element analysis (FEA); Thermal analysis 


\section{Introduction}

2.5D angle-interlock woven fabric composite (2.5DAWC) is a novel and special type of textile structural composites, having attracted increasing interesting in various fields, such as aircraft, high performance automotive, maritime craft and civil engineering, owing to its easy processing in a tradition loom. 2.5DAWC consists of undulated warp yarns and non-crimp weft yarns impregnated in matrix, showing better delamination resistance, damage tolerance, high impact resistance, higher tensile strain-to-failure values and higher interlaminar fracture toughness compared with traditional 2D laminated composites. This kind of 3D composite shows a wide application prospect. Although the mechanical properties of 2.5D AWC have been widely studied, the thermal conductive properties of it have never been reported. Because in most case composite materials are used in dramatic temperature changing environment, thermal stress concentration caused by bad heat dissipation is also the main reason of composites fatigue and failure. Therefore, more and more requirements are needed to reveal the thermal conductive behaviors of complicate 3D textile structural composite materials.

Many studies indicated that the thermal conductivity of complicated structural composites from 2D reinforced to 3D structural composites. As for the thermal conductive research of $2 \mathrm{D}$ plain woven composites, the early researchers have made a great effort. Dasgupta $[1,2]$ used a homogenization technique to study the thermal conductivity of plain weave composites. Afterward, Ning and Chou $[3,4]$ presented the closed-form solutions based on thermal-electrical analogy to predict the in-plane and through-thickness effective thermal conductivities of plain woven composites. Later, Bhattacharjee et.al [5,6] developed a mathematical model based on the principles of heat transfer to predict the thermal resistance of fabrics. Siddiqui et.al $[7,8]$ began to use the finite element method (FEM) systematically to predict the effective thermal conductivity and thermal resistance of woven composite. As the 3D textile structural composites gradually replacing the 2D laminated structure have attracted more and more attention, the focus of thermal conductive research is also turned to 3D structure. For example, Gowayed [9] firstly investigated the thermal conductivity 3D textile composites and also discussed the effect of fiber type and fiber volume fraction on its thermal conductivity. And for 3D knitted structure, Hasani et.al [10] applied the finite element method to analyze the heat transfer behavior of plain interlock weft knitted 
fabrics. For 3D orthogonal woven structure, Ai et.al [11] demonstrated the average in-plane and out-of-plane thermal conductivities of 3D orthogonal woven $\mathrm{C} / \mathrm{C}$ composites combining with experimental measurement and three dimensional steady state heat transfer finite element analysis. They carried out the finite element analysis at two distinct length scales, i.e. micro-scale and meso-scale and draw a conclusion that the multi-scale models provided an efficient approach to predict the thermal conductivities of 3D textile materials. For 3D braided structure, several researchers have made significant progresses. For example, Jiang et.al [12] chose a factual and reasonable micro-structural model of braided composites to improve the calculating precision of thermal property of 3D braided composites by FEM. Jiang et.al [13] presented a modified FEM to investigate the thermal conductivity and temperature distribution of 3D braided composites. And they began to study the temperature distribution in a complex structure. Tao et.al [14-16] used FEM to systematically study the thermal conductivity of plain woven composited and 3D braided composites by different unit cell scales. They also used the lattice Boltzmann method to predict the thermal conductivity of 3D braided composites. For non-woven structure, Lee et.al [17] used the analogy between the diffusion of heat and electrical charge to predict the thermal conductivities of multiaxial non-woven composites. And for stitched structure, Kumar et.al [18] investigated the thermal diffusivity of 3D-stitched fiber architecture composites in in-plane and through-thickness directions.

Above is the present research status about the thermal conductive properties of complicated structural composites, from which we can make the following conclusions. The finite element analysis method is widely applied in the thermal conduction research of complicated structural composites. The thermal conductivities of 2D plain woven laminated, 3D orthogonal woven, 3D weft knitted, 3D braided, 3D multiaxial non-woven and 3D stitched have already been studied, however, the thermal conductive research of 2.5D angle-interlock woven composites has never been reported. In addition, few researchers can effectively reveal and characterize the temperature distribution and heat flux transitting path of complicated structural composites. Moreover, the internal structural defects, e.g. voids, are seldom considered in the present thermal conductive research.

In order to solve the above problems existed in the present thermal conductive research of complicated structural composites, this paper investigated the thermal conductive properties of 2.5DAWC both from 
experimental and finite element analyses (FEA). Three representative volume element (RVE) were selected from yarns, matrix and composites to gradually obtain the thermal conductivity of $2.5 \mathrm{DAWC}$. The effects of internal voids and interface contact thermal rsistance between fiber and resin on the thermal conductivities of 2.5DAWC are also considered and analyzed. The temperature distribution and heat flux transmitting path of 2.5DAWC are revealed from FEA. This article helps for further understanding the thermal conductive hehaviors of complicated structural composites, and also contributes to investigating the thermal-mechanical properties of 2.5DAWC.

\section{Experimental procedure}

\subsection{Materials preparation}

The 2.5D angle-interlock woven fabric (2.5DAWF) with layer to layer angle-interlock structure was fabricated by Torayca carbon fiber tows (T300-6k for warp, T700-12k for weft, manufactured by Toray, Japan ). Table 1 shows the specifications of the 2.5DAWF. Epoxy resin (JA-02C) from Jiafa Chemical Co., China, was used to impregnate the AWF with the assistant of vacuum assisted resin transfer method (VARTM) process. The epoxy resin was first vacuumed to remove the air bubbles before being injectd into the preforms. As shown in Fig. 1, double vacuum bags were used to ensure a stable pressure imposing to fabric during the process of curing. The resin epoxy was injected into the first bags from the two injection ports at the same time, and outflowed from the center vent. The side vent just extracted air from the second vacuum bag and didn't inject resin. The curing temperature was $90^{\circ} \mathrm{C}$ for $2 \mathrm{~h}, 110^{\circ} \mathrm{C}$ for $1 \mathrm{~h}$ and finally $130^{\circ} \mathrm{C}$ for $4 \mathrm{~h}$. The final specimens were removed from the oven after cooling for $12 \mathrm{~h}$, and then cut into the rectangle shape with size $50 \times 50 \times 6 \mathrm{~mm}$ by means of high-pressure water jet. The tests were conducted in standard conditions at a room temperature of $25 \pm 2^{\circ} \mathrm{C}$ and $65 \pm 2 \%$ relative humidity to ensure its reliability.

\subsection{Experimental setup}

All the testing works were conducted in a self-designed apparatus [19] which has already been calibrated by standard aluminum (5052) specimens with known thermal conductivity $138 \mathrm{~W} \mathrm{~m}^{-1} \mathrm{~K}^{-1}$. The range of measuring errors was kept within 5\%, which demonstrated the reliability of this apparatus. The apparatus mainly consists of three parts, i.e. thermal insulation, temperature controlling system and signal acquisition system. An adiabatic chamber for measuring the thermal conductivity of the 
2.5DAWC was composed of foam insulation boards and alumina fiber plates, which can effectively prevent the heat from diffusing to outside air. The binding sites of foam insulation boards and alumina fiber plates were fixed with heat insulated adhesive sealing tape. The temperature controlling system could make the temperature of heat source stable with $-1-6{ }^{\circ} \mathrm{Cdeviation}$, which could provide a stable thermal gradient to the specimens. The signal acquisition system could record the temperature changes of thermocouples every one minute, which can effectively record the temperature change in the process of thermal conduction. It is assumed that when the temperature variation satisfied $\triangle \mathrm{T} \leq 0.1^{\circ} \mathrm{C}$, the status of thermal conductive equilibrium was achieved and then the experiment was finished.

\subsection{Thermal conductive measurement}

In this paper, all the experimental methods were based on the comparative longitude heat flow method [20] and steady-state plane source method [21]. In these methods, the specimens with unknown thermal conductivity are compressed between the known reference specimens and heat flux passing through the measuremnet unit as a temperature difference is created between the two sides of the unit. The thermal conductivities of the specimen and the reference specimens are inversely proportional to their thermal gradients. The tests are also in accordance with ASTM E-1530 standard. Considering carbon fiber have obvious anisotropic thermal conductive characteristics, the thermal conductive measurement should be conducted along different directions. Fig. 2 shows the definitions of the in-plane and out-of-plane directions. The aluminum 5052 plates with the same size as the composite coupons were selected for auxiliary measuring the thermal conductivity of all the fabricated composites. Figs. 3 (a) and (b) shows the experiemntal process of the thermal conductive measurement along the out-of-plane direction and in-plane direction, respectively. The figures 1-6 in Fig. 3 refer to the composite coupons, aluminum 5052 plate, heating aluminum plate, alumina fiber insulation board, lower foam board and upper cover foam board, respectively. The reference aluminum plates were tightly glued on the surface of composite coupons. Just as the distributions of the hollow red circles in Fig. 3, the K-type thermocouples with the prescribed spacings were embedded into the pre-built holes. The embedding depth of them was set as $3 \mathrm{~mm}$. The alumina fiber insulation boards were used to cover the side faces of pecimens to make sure these faces under adiabatic conditions. The assembling processes of this apparatus are as follows: firstly the heating aluminum plate was embedded in the middle of the lower foam board, and then the composite coupons were fixed on the top of this board by aluminum foil tapes, 
and finally the upper foam board covered the composite coupons and the lower foam board. It is assumed that no gap existed in all the cotact areas because the epoxy resin adhesive have filled into these gaps. By adding the thermal grease HT-GY 260 (thermal conductivity $>2.8 \mathrm{~W} \mathrm{~m}^{-1} \mathrm{~K}^{-1}$ ), it is also assumed that no contact thermal resistance existed between the specimens and the heating plate. When the steady state is reached, the temperature slope is linear along the reference specimen and its thickness. Knowing the thermal conductivity of reference aluminum plate and the temperature differences between the adjacent thermocouples, the heat flux of the hot and cold sides can be calculated with one dimensional Fourier's heat conduction law. From the average of the heat fluxs, the thermal conductivity of the composite coupon can be calculated by:

$$
\lambda_{C}=\lambda_{r} \frac{A_{r} \cdot \frac{1}{n} \sum_{i=1}^{n} \frac{\Delta T_{r i}}{x_{r i}}}{A_{C} \cdot \frac{1}{n} \sum_{i=1}^{n} \frac{\Delta T_{C i}}{x_{C i}}}
$$

where $\lambda$ is the thermal conductivity, $A$ is the cross-section, $\Delta T_{i}$ is the temperature differences and $x_{i}$ is the distance between adjacent thermocouples. $n$ is the total number of thermocouples. The subscript $r$ and $C$ refer to the reference aluminum plate and the composite coupon, respectively. The purpose of this paper is to measure the thermal conductivity of $2.5 \mathrm{DAWC}$ at room temperature, therefore, the temperature differences between the hot and cold sides were controlled within $10{ }^{\circ} \mathrm{C}$. Each test was repeated at least three times both along in-plane and out-of-plane directions.

\section{Finite element analyses}

\subsection{Microstructure model of 2.5DAWC}

In order to better predict the thermal conductive properties of 2.5DAWC, it is necessary to establish a real geometric microstructural model of it. Based on the experimenal observations of optical microscope (Eclipse E200, Japan) and the actual distance measurement by image processing software ImageJ, the basic structural parameters of 2.5DAWC can be measured. As shown in Fig. 4, the 2.5DAWC construction is composed of three parts, i.e. the resin matrix, warp and weft yarns. The yarn system presents a layer-to-layer angle-interlocking structure where the weft yarns are almost straight. The two adjacent undulated warp yarns in a single layer display a converse undulation form to ensure the two adjacent layers of non-crimp weft yarns are held together to form a stable and integrated woven construction. The sparing parts except yarns are filled with the resin matrix. By microscopic 
observation and our previous work [22], the racetrack shape was selected as the cross-sections of weft and warp yarns. The axial line of the weft yarn was assumed to be straight, while the center line of warp yarn is composed of two parts: an arc in the interlock edge considering the tightening effect of yarns, and a straight line in the rest parts. All the geometric microstructural, including the length and width of warp and weft yarns, the gap between adjacent warp and weft yarns, the horizontal and the vertical spacing between adjacent warp yarns or adjacent weft yarns, were measured by ImageJ software from several photographs at least ten times. The average values calculated from these data are shown in Fig. 5. According to these parameters, the volume fraction of yarns in composites $v_{y}$ can be derived as:

$v_{y}=\frac{V_{y}}{V_{C}}$

where $V_{y}$ is the volume of weaving yarns, which can be warp and weft. $V_{C}$ is the volume of the whole composites. In this paper, the $v_{y}$ is $68.18 \%$, where the corresponding volume fractions of warp and weft yarns are $32.4 \%$ and $35.78 \%$, respectively.

By assuming the carbon fibers are evenly distributed and arranged parallel to each other in yarns, the volume fraction of fibers in yarns $\delta_{f}$ can be calculated just as follows:

$$
\delta_{f}=\frac{\pi d^{2} \cdot n_{f} l_{f}}{4 S_{y} l_{y}}
$$

where $d$ is the diameter of a single carbon fiber, $n_{f}$ is the total number of fibers in a single yarn, $S_{y}$ is the cross sectional area of a single yarn. $l_{f}$ is the length of fiber in unit cell, $l_{y}$ is the length of yarn in unit cell, where $l_{f}$ can be equal to $l_{y}$ by assumption. The values of these parameters and $\delta_{f}$ are listed in Table 2.

The volume fraction of fibers in composites $v_{f}$ can be further calculated by combining the volume fraction $\delta_{f}$ and $v_{y}$, which can be written as:

$$
v_{f}=\frac{\delta_{f}}{v_{y}}
$$

As we all know, there are some voids existing in the composite materials during their curing process. 
The so-called voids, i.e. the motionless air, have a significant influence in the thermal conduction of composites. Fig. 6 shows the scanning electron microscope (SEM TM-3000, Japan) photographs of the voids in 2.5DAWC. Considering the actual difficulties in measuing the volume fraction of voids, it is assumed that the average area ratio between the voids and the whole photograph calculating from several SEM photographs are treated as the volume fraction of voids in composites. This volume fraction of voids in composites is also considered in FEA, which can be seen in section 3.2.

Table 3 shows the volume fraction of fibers, resin matrix and voids in composites both from experimental and FEA. The actual volume fractions of warp and weft yarns in composites were measured by the combustion method in muffle furnace (temperature is set as $600{ }^{\circ} \mathrm{C}$ and the burning time is more than $6 \mathrm{~h}$ ). By removing the contents of yarns and voids, the rest part is the resin matrix.

\subsection{Multi-scale models}

In this paper, the FEA were operated on the commercial available finite element software package ABAQUS/Standard (ver. 6.12) under the environment of operation system LINUX. All the geometrical models were established in ABAQUS directly. The 2.5DAWC is composed of resin matrix, warp and weft yarns. Therefore, before the calculation of 2.5DAWC, the thermal conductivities of woven yarns and matrix have to be obtained firstly. In this paper, multi-scale finite element models were adopted to gradually calculate the thermal conductivity of 2.5DAWC. As shown in Fig. 7, two micro-scale models i.e. the matrix-voids RVE with square structure and the fiber-matrix (yarn) RVE with rectangular structure in red rectangular boxes were selected to calculate the thermal conductivities of resin matix and yarns, respectively. The meso-scale model i.e. the RVE of 2.5DAWC in blue rectangular box was used to obtain the thermal conductivity of composites based on the two micro-scale models.

Firstly the matrix-voids RVE can be regarded as the assembly of resin matrix and voids. Its thermal physcial properties can be obtained by the basic thermal physical parameters of matrix and voids. In FEA it is assumed that the voids only exist in the resin areas and uniformly distributed in them. In order to analyze the effect of voids on the final thermal conducive behaviors, different volume fractions of voids i.e. $1.2 \%, 5 \%$ and $10 \%$ were selected in FEA. The properties of matrix-voids RVE were then inputted into the models of yarns and composites, respectively. The yarn i.e. fiber-matrix RVE is treated as unidirectional (UD) fiber reinforced composite composed of fibers, matrix-voids RVE, and the interface between them. And the final thermal conductivity of $2.5 \mathrm{DAWC}$ can be further calculated 
by the matrix-voids RVE and the fiber-matrix RVE. The basic thermal physical properties of fibers, epoxy resin and voids are listed in Table 4.

\subsection{Governing equations}

The FEA of 2.5DAWC were run for steady-state conditions to obtain temperature and heat flux throughout the model. The governing equations is described as:

$k_{x x} \frac{\partial^{2} T}{\partial x^{2}}+k_{y y} \frac{\partial^{2} T}{\partial y^{2}}+k_{z z} \frac{\partial^{2} T}{\partial z^{2}}+\left(k_{x y}+k_{y x}\right) \frac{\partial^{2} T}{\partial x \partial y}+\left(k_{x z}+k_{z x}\right) \frac{\partial^{2} T}{\partial x \partial z}+\left(k_{y z}+k_{z y}\right) \frac{\partial^{2} T}{\partial y \partial z}=0$

where $T$ is the temperature, $k_{x x}$, etc. are thermal conductivity, which can be regarded as transversely isotropic for carbon fiber and isotropic for resin matrix. The constitutive relationships used in FEA are the same as Fourier's heat conduction law and can be defined as:

$q=-k \nabla T$

where $q, k$ and $\nabla T$ are heat flux density, thermal conductivity and temperature gradient, respectively.

\subsection{Boundary conditions}

Based on the analyses of the above models, different boundary conditions were applied to FEA. Because the micro-scale RVE and the four sides of meso-scale RVE have the characteristic of periodic structural distribution, the periodical temperature boundary conditions (PBCs) [23] are used to obtain a reasonable and uniform temperature and heat flux distributions. The PBCs can ensure that each RVE in the composite has the same loading mode and there is no separation or overlap between the neighboring RVEs. The location and numbering of the faces, edges and vertices in RVE are shown in Fig. 8(a). In the cubic unit-cell with periodicity in all the three directions, $\xi-\xi^{\prime}, \eta-\eta^{\prime}$ and $\zeta-\zeta^{\prime}$ are pairs of periodic nodes locating on the opposite parallel boundary surfaces of the unit-cell and the lines linking them are parallel to the three coordinate axes, respectively. According to the applying methods of PBCs in ABAQUS user manual [24], the temperature of the cubic cell can be constraint by setting the point $A$ as a datum mark to be fixed, point $B$ to be constraint in $z$ direction and point $D$ to be constraint in $x$ and $z$ directions. Taking the corner points $A^{\prime}, B$ and $D$ as the master nodes, 
the equations of PBCs are summarized as follows:

- Faces

$T^{\xi^{\prime}}-T^{\xi}-T^{D}=0, \quad T^{\eta^{\prime}}-T^{\eta}-T^{B}=0, \quad T^{\zeta^{\prime}}-T^{\zeta}-T^{A^{\prime}}=0$

- Edges

$T^{C^{\prime} D^{\prime}}-T^{C D}-T^{A^{\prime}}=0, \quad T^{C^{\prime} D^{\prime}}-T^{A^{\prime} B^{\prime}}-T^{D}=0, \quad T^{A^{\prime} B^{\prime}}-T^{A B}-T^{A^{\prime}}=0$

$T^{C^{\prime} C}-T^{D D^{\prime}}-T^{B}=0, \quad T^{C^{\prime} C}-T^{B B^{\prime}}-T^{D}=0, T^{B B^{\prime}}-T^{A A^{\prime}}-T^{B}=0$

$T^{B C}-T^{A D}-T^{B}=0, \quad T^{B^{\prime} C^{\prime}}-T^{B C}-T^{A^{\prime}}=0, \quad T^{B^{\prime} C^{\prime}}-T^{A^{\prime} D^{\prime}}-T^{B}=0$

- Vertices

$T^{C}-T^{D}-T^{B}=0, T^{D}-T^{D^{\prime}}-T^{A^{\prime}}=0, T^{B^{\prime}}-T^{A^{\prime}}-T^{B}=0, T^{C^{\prime}}-T^{C}-T^{A^{\prime}}=0$

where $T$ is the temperature, $A$, etc. are the terminal vertexs in the RVE. The degree of freedom for heat conduction module is 11 in ABAQUS. In these linear multi-point constraint equations, the temperature of master node is equal to that of the face or edge where the master node is contained. Therefore, only by applying the thermal gradient to the master node, the face or the edge will be also under the same thermal loading. It should be noted that the meshes on the two opposite vertices, edges and faces of the unit-cell should be same for applying the above equations. The present periodical temperature boundary conditions have been justified by applying thermal gradient to opposite nodes both in micro-scale and meso-scale RVE, whose error was controlled within $\pm 5 \%$.

The upper and lower faces of meso-scale model are the real surfaces of composite, therefore, there is no need to impose periodic boundary conditions. Fig. 8(b) shows the boundary conditions for the thermal conductive analysis of the out-of-plane direction. The thermal gradient goes through the hot source to the hot sink to provide steady heat flux for passing the composites. The other surfaces were set as adiabatic.

\subsection{Meshing}

Considering the complexity of geometrical structure and the demanding for high quality of grid, the powerful finite element meshing software HyperMesh (ver. 12.0) was used. The process of meshing is as follows: through reasonable segmentation, the grid of resin were firstly created, and then the nodes of resin in the contact regions were projected onto yarns, the grid of yarns were generated based on these projected nodes. Because the results of thermal conduction were greatly influenced by the mesh 
sizes and mesh quality, this paper attempted different mesh sizes in HyperMesh and verified the mesh quality in the mesh module of ABAQUS with the purpose of reducing the number of warning grid as well as obtaining suitable total number of mesh. The present mesh sizes of the matrix-voids RVE, fiber-matrix RVE and composite unit cell were set as $0.02,0.02$ and $0.2 \mathrm{~mm}$, respectively, as a result of reducing computational cost and ensuring the computation quality. The detailed meshing schemes of multi-scale models are shown in Table 5, including the size of model, the number of nodes in faces and edges, the type, shape and number of elements, and the way of nodes' constraint.

\section{Results and discussions}

\subsection{Experimental supplementary verification}

In this paper, in order to verify the reliability of thermal conductive experiment using the self-designed apparatus, the thermal conductivity testing instrument DZDR-S based on transient plane source (TPS) technique [25] was also used. Fig. 9 shows the testing process. The sensor, typically double concentric ring with multiple spirals of nickel wire, is sealed between two Kapton films for electrical insulation. The constant current is passed through the sensor at the beginning of the experiment. The temperature increase in sensor is monitored by resistance charge. The thermal property of the sensor at various temperatures is a known quantity. The conductivity of a sample can then be obtained by solving the differential equation of conduction [26]. In addition, because the sensor of the DZDR-S instrument has a certain size, the radius of the specimen must be more than $5 \mathrm{~mm}$ to cover the sensor. However, the thickness of the specimen is $6 \mathrm{~mm}$, which is less than the minimum size the instrument needs. Therefore, the thermal conductive measurement in in-plane direction should use this self-designed device, whose reliablity will be verified by the DZDR-S.

In section 2.3 , the thermal conductive measuring method by self-made apparatus and the calculating formula of thermal conductivity have been introduced. The average temperatures of these embedding thermocouples are plotted in Fig.10. All the curves show similar features. The temperatures drop slowly in the aluminum parts, while it drops faster in the parts of specimens. Because the thermal conductivity of aluminum is much higher than that of composites, higher thermal conductivity is more benefit for the transfer and diffusion of temperature. The thermal conductivity of experimental can be calculated by Eq.(1). The comparisons between the results from DZDR-S and self-designed device are listed in Table 6. The good agreement is shown between them, indicating the reliablity of thermal 
conductivities measured by the self-designed apparatus.

\subsection{Micro-scale RVE}

Fig. 11 shows the heat flux and temperature distribution of the matrix-voids RVE. It is obvious that the model with more voids' content has more uneven distribution of heat flux and temperature, voids play obvious role in preventing heat flux transmission. Because the resin matix and voids are all isotropic materials, the properties of their combination matrix-voids RVE can also be regarded as isotropic. Therefore, only applying thermal gradient to one direction can enough characterize the thermal conductive properties of matrix-voids RVE. According to the thermal properties of resin matix and voids (still air), the basic thermal physical parameters of matrix-voids RVE with different voids' contents can be obtained. The density and specific heat at constant pressure of the mixed matrix-voids RVE can be written as:

$$
\begin{aligned}
& \rho_{m}=V_{i} \rho_{i}+V_{j} \rho_{j} \\
& C_{p m}=M_{i} C_{p i}+M_{j} C_{p j}
\end{aligned}
$$

where $\rho$ is the density, $C_{p}$ is the specific heat at constant pressure, $V$ and $M$ are the volume fraction and the mass fraction of its corresponding components, respectively. The subscripts $i$ and $j$ are the constituent parts of the mixed system.

The fiber-matrix RVE i.e. unit cell model of yarn contains one and four $1 / 4$ fibers embedded in the matrix-voids system. Because the thermal conductivities of carbon fiber have obvious anisotropic characteristics, the thermal gradient should be applied to different directions in the fiber-matrix RVE. However, the orientation direction of yarn does not agree with the global coordinates direction of composites, therefore, it's worth noting that the orientation defnition of the yarn's model in the local coordinate system. Fig. 12 shows the local coordinate orientation definition of yarn. The three coordinate directions in local coordinate system are set as 1,2 and 3, where 11 is the axial direction and 22,33 are the radial directions of yarn. By applying thermal gradient to the corresponding nodes in the above PBCs, the heat flux and temperature distributions of yarn system can be easly obtained, just as shown in Fig. 13. Because the thermal gradient applied in the directions $i i(i=1,2,3)$ can hardly extend to the shear directions $i j(i, j=1,2,3 ; i \neq j$ ), the thermal conductivities of the shear 
directions $k_{i j}(i, j=1,2,3 ; i \neq j)$ are close to zero.

It is well known that the contact thermal property existed at the interface is an important factor influencing the final thermal conductive results. In composites, the contact region mainly exist between fiber and resin, whose effects we can't ignored. While other contact regions including yarn and resin matirx, matrix and voids, were not considered in this paper. Considering the thermal contact resistance with no pressure and at room temperature between fiber and resin in reference [27], it is assumed that the interface contact thermal conductivity between them was $1.5 \times 10^{5} \mathrm{~W} \mathrm{~m}^{-2} \mathrm{~K}^{-1}$. This paper extracted a single fiber embedded matrix model to study the effects of interface thermal resistance. Fig. 14 shows the isothermal contours and heat flux lines distribution of this model under steady-state heat transfer analysis in COMSOL (ver. 4.4). The interface thermal resistance has no obvious effects on the thermal conductive behaviors of this model, only showing the slight deflection of heat flow lines and small decrease of the temperatures in the interfaces. In the following analyses, we will make a comprehensive consideration about the effects of having interface and no interface on the final thermal conductive results of 2.5DAWC. The thermal properties of matrix-voids RVE and fiber-matrix RVE (yarn model) under different voids' content are shown in Table 7.

\subsection{Meso-scale RVE}

The meso-scale RVE, i.e. unit cell model of composite, formed by fiber-matirx (yarn) system embedded in the matrix-voids system, was used to analyze the final thermal conductive behaviors of 2.5DAWC. In the global coordinate system, the three coorainate directions $\mathrm{xx}, \mathrm{zz}$ and yy represent the directions of the warp, weft and through-thickness of the composite, respectively. The thermal gradients were applied to these directions according to the experimental results. Because of the periodicity of the meso-scale RVE in the warp and weft directions, the temperatures of hot source were only applied to the master points $A^{\prime}$ and $B$ by $\mathrm{PBCs}$, which were $35^{\circ} \mathrm{C}$ based on experimental. Considering the convenience of PBCs applied in FEA, the temperture of the fixed point $A$ i.e. the hot sink was set to be $0{ }^{\circ} \mathrm{C}$. This paper only considers the thermal coductivity of $2.5 \mathrm{DAWC}$ at room temperature, therefore, the biggest fluctuation range of temperatures in FEA was controlled between $0{ }^{\circ} \mathrm{C}$ and $35^{\circ} \mathrm{C}$, while for experiment the range is $25-35^{\circ} \mathrm{C}$. It is assumed that the thermal conduction properties are the same in these two temperature ranges $\left(0-35^{\circ} \mathrm{C}\right.$ and $\left.25-35^{\circ} \mathrm{C}\right)$, considering no obvious 
effect occurred in $0-25^{\circ} \mathrm{C}$.

Fig. 15 shows the distributions of heat flux in different directions by applying thermal gradient in the warp direction. Accordingly, its isothermal contour maps of meso-scale RVE are shown in Fig. 16. The obvious inhomogeneity of the heat flux distribution can be found, presenting the heat fluxs are mainly distributed in the regions of weft yarns, later in the warp yarns and finally in the resin matrix. This is because the heat fluxs tend to be distributed in the material with higher thermal conductivity and the weft yarns have the highest thermal conductivities in this hybrid material system. Through the decomposition of heat flux in three coordinate directions, the warp direction i.e. along the direction of thermal gradient has more heat flux distribution than the weft and thickness directions. The heat flux is mainly along the direction of thermal gradient and almost no transmits to other directions, resulting in the heat fluxes are very small in non-thermal gradient directions. Fig. 16 displays its corresponding steady state temperature distributions of meso-scale RVE by applying thermal gradient along warp direction. The temperature distribution of meso-scale RVE shows a strong correlation with the spacing distribution of yarns and the thermal conductivities of constitute materials. Because the axial thermal conductivity of warp yarns are higher than the radial thermal conductivity of weft yarns and that of resin, materials with high thermal conductivity are more benefit for temperature transmission, the temperature transmission distance of warp yarns is obvious longer than that of weft yarns and resin. In addition, the temperatures of overlapping areas are higher than those of other areas. This is due to the heat exchange occuring in the thermal conduction process, and the heat exchange ability will becomes stronger between materials with high thermal conductivity. And also for this reason, the temperature distribution of the resin matrix is basically consistent with that of yarns' system. The fillers e.g. fibers can make the thermal conduction ability of matrix materials greatly improved.

Fig. 17 shows the temperature and heat flux distributions along the weft and through-thickness directions. Different from the warp direction by applying hot source on point $B$, the weft direction use the point $A^{\prime}$ as hot source and the point $A$ as hot sink to apply thermal gradient. The similar heat flux and temperature distributions of thermal conduction in warp direction are observed in weft direction, except that the temperature transmission distance of weft yarns is longer than that of warp yarns and resin. Because in this direction, the the axial thermal conductivity of weft yarns are higher than the radial thermal conductivity of warp yarns and that of resin. Different from thermal conduction 
in warp and weft directions, the through-thickness direction has the hot source and hot sink on the upper and lower surface of the meso-scale model. The heat fluxes are also mainly distributed in the yarns' area. However, the temperature distribution is relatively uniform, considering the radial thermal conductivity of warp and weft yarns is approximately equal.

Fig. 18 plots the average temperatures of meso-scale RVE at different locations, for the sake of describing the overall temperatures of the different components in 2.5DAWC. It can be found that the average temperatures of the resin, warp and weft yarns are very similar, especially in the thickness direction. This is due to the steady-state thermal conduction method was selected to reduce the computing time, the temperatures of these curves are the final thermal equilibrium temperature. However, the cyclical fluctuations of temperatures are found on the warp and weft yarns in the warp applying direction, and also found on the warp yarns in the weft applying direction. There are mainly two reasons explaining these phenomena: on the one hand, there are several oblique line segments existing in the warp yarn, which will greatly reduce the thermal conduction ability of the whole composites. If the oblique angle is assumed as $\theta$, just as shown in Fig. 5, the thermal conductivity of the oblique line segments $k_{\theta}$ can be expressed as [19]:

$k_{\theta}=k_{x x} \cos ^{2} \theta+k_{y y} \sin ^{2} \theta$

where $k_{x x}$ and $k_{y y}$ are thermal conductivities of $\mathrm{xx}$ and yy directions, respectively. $k_{\theta}$ is far less than $k_{x x}$ resulting in the declining ability of thermal conduction. On the other hand, the weft yarns in warp direction and the warp yarns in weft direction are discontinuity, the discontinuous area i.e. the resin matrix will make the temperature drop considerably. This is why the curves show high and low.

In order to further illustrate these phenomena, the temperatures and heat flux densities of points $A$ and $B$ were extrated to calculate the thermal conductivity along warp direction. It is found that the thermal conductivity of $\mathrm{xx}$ direction is much larger than that of other directions. Moreover, the thermal conductivities of yy and zz directions are almost zero. The similar results along weft direction can also be obtained by extrating temperatures and heat flux densities of points $A$ and $A^{\prime}$. The thermal conductivity of $\mathrm{zz}$ direction is much larger than that of $\mathrm{xx}$ and $\mathrm{yy}$ directions, whose thermal conductivities are close to zero. By extracting separately the average temperatures and average heat flux densities of the upper and lower surface of meso-scale RVE, the thermal conductivity of 
through-thickness direction can be calculated. It represents the thermal conductivity of yy direction is larger than that of $\mathrm{xx}$ and $\mathrm{zz}$ directions, whose thermal conductivities are also close to zero. From these results, it can be concluded that the heat fluxes are mainly concentrated in the direction of applying thermal gradient, temperature distribution depends on yarns' orientation and thermal conductivity of components. As for the thermal conductivity, the 2.5DAWC can be regarded as transversely thermal isotropic material.

\subsection{Transient temperature distribution}

The steady-state thermal conduction can't show the transient temperature distribution of each component in 2.5DAWC. However, the transient temperature distribution is an improtant method to characterize the thermal conduction abilities of different materials. Fig. 19 displays the transient temperature distributions both along the warp and weft directions. The transient (time-dependent) thermal conduction analyses were also conducted under COMSOL with $1 \mathrm{~s}$ thermal conduction time. The thermal conduction along the weft direction in Fig. 19(a) shows that the temperature transmission rate of weft yarns are obvious faster than that of warp yarns and resin. The similar conclusion can also be found along warp direction in Fig. 19(b), which shows that the temperature transmission rate of warp yarns are obvious faster than that of weft yarns and resin. These phenomena can be explained by that material with higher thermal conductivity has obvious faster temperature transmission rate, accordingly, the axial tempeature transmission rate will be faster than that of radial.

\subsection{Effects of voids and interface thermal contact resistance}

This paper also consider about the effects of voids and interace thermal contact resistance between fiber and matrix on the final thermal conductivity of 2.5DAWC. Three types of matrix-voids models with different voids volume fraction were used to calculated the thermal properties of matrix-voids. Then the thermal properties of matrix-voids RVE were inputted into yarn and composite models. The thermal contact resistance was only considered at the interface between fiber and resin when the voids volume fraction was $1.2 \%$. The final thermal conductivities of $2.5 \mathrm{DAWC}$ are listed in Table 8 . It is found that the FEA results show a good agreement with the experimantal, the thermal conductivities with interface are closer to the experimantal than that of without interface. With the increase of voids volume fraction of composites, the overall thermal conductivity of composites presents the obvious 
declining trend.

\section{Conclusions}

The thermal conduction properties of 2.5DAWC along warp, weft and through-thickness directions were investigated both from experimental and FEA. The effects of voids and interface thermal contact resistance on the thermal conductivity are also considered. Based on the results of the thermal conductivity measurements and the numerical analysis, the following conclusions can be reached:

1. The self-designed thermal conduction apparatus can effectively measure the thermal conductivity of 2.5DAWC. This apparatus can be also used for the thermal conductivity measurement of other complicate structural composites.

2. The multi-scale methods of FEA can well predict the thermal conduction behaviors of 2.5DAWC, which keeps a good agreement with the experimental results. The thermal conductivities along different applying directions of 2.5DAWC strongly depend on the thermal conductivity of its components.

3. The temperature ans heat flux distributions are mainly influenced by yarns' location, orientation and thermal conductivity. Because of the heat exchange, the material with higher thermal conductivity will transfer heat to that of lower thermal conductivity, and that's why the temperatures of resin part are greatly elevated.

4. It is a good method to simplify the effects of voids and interface thermal contact resistance into the micro-scale models. Both the voids and the interface thermal contact resistance can reduce the final thermal conductivity of composites, and the influence of voids is more apparent. The thermal conductivities are closer to the experimental by considering the effects of voids and interface thermal contact resistance.

\section{Acknowledgements}

The authors acknowledge the financial supports from the Chang Jiang Scholars Program and National Science Foundation of China (Grant Number 11272087 and 11572085). The financial supports from State Key Laboratory for Modification of Chemical Fibers and Polymer Materials, Donghua University, Foundation for the Fok Ying-Tong Education Foundation (Grant No. 141070), "Shu Guang" project (Grant No. 14SG31) supported by Shanghai Municipal Education Commission and Shanghai Education Development Foundation, the Fundamental Research Funds for the Central 
Universities of China, the Fundamental Research Funds for the Central Universities and Fund of National Engineering and Research Center for Commercial Aircraft Manufacturing (Project SAMC14-JS-15-048 and Project SAMC14-JS-15-049) and DHU Distinguished Young Professor

Program are also gratefully acknowledged.

\section{References}

[1] Dasgupta A, Agarwal RK. Orthotropic thermal conductivity of plain-weave fabric composites using a homogenization technique. Journal of Composite Materials. 1992,26(18):2736-2758.

[2] Dasgupta A, Agarwal R, Bhandarkar S. Three-dimensional modeling of woven-fabric composites for effective thermo-mechanical and thermal properties. Composites Science and Technology. 1996,56(3):209-223.

[3] Ning Q-G, Chou T-W. Closed-form solutions of the in-plane effective thermal conductivities of woven-fabric composites. Composites Science and Technology. 1995,55(1):41-48.

[4] Ning Q-G, Chou T-W. A general analytical model for predicting the transverse effective thermal conductivities of woven fabric composites. Composites Part A: Applied Science and Manufacturing. 1998,29(3):315-322.

[5] Bhattacharjee D, Kothari VK. Heat transfer through woven textiles. International Journal of Heat and Mass Transfer. 2009,52(7-8):2155-2160.

[6] Bhattacharjee D, Kothari V. A theoretical model to predict the thermal resistance of plain woven fabrics. Indian Journal of Fibre \& Textile Research. 2005,30(3):252.

[7] Siddiqui MOR, Sun D. Finite element analysis of thermal conductivity and thermal resistance behaviour of woven fabric. Computational Materials Science. 2013,75:45-51.

[8] Siddiqui MOR, Sun D. Computational analysis of effective thermal conductivity of microencapsulated phase change material coated composite fabrics. Journal of Composite Materials. 2014,49(19):2337-2348.

[9] Gowayed Y, Hwang J-C. Thermal conductivity of composite materials made from plain weaves and 3-D weaves. Composites Engineering. 1995,5(9):1177-1186.

[10] Hasani H, Ajeli S, Nouri N. Modeling of heat transfer for interlock knitted fabric using finite element method. Indian Journal of Fibre \& Textile Research. 2014,38(4):415-419.

[11] Shigang A, Rujie H, Yongmao P. A Numerical Study on the Thermal Conductivity of 3D Woven C/C Composites at High Temperature. Applied Composite Materials. 2015,22(6):823-835.

[12] Liu Z, Zhang H, Lu Z, Li D. Investigation on the Thermal Conductivity of 3-Dimensional and 4-Directional Braided Composites. Chinese Journal of Aeronautics. 2007,20(4):327-331.

[13] Jiang LL, Xu GD, Cheng S, Lu XM, Zeng T. Predicting the thermal conductivity and temperature distribution in 3D braided composites. Composite Structures. 2014,108(1):578-583.

[14] Fang W-Z, Chen L, Gou J-J, Tao W-Q. Predictions of effective thermal conductivities for three-dimensional four-directional braided composites using the lattice Boltzmann method. International Journal of Heat and Mass Transfer. 2016,92:120-130.

[15] Gou J-J, Dai Y-J, Li S, Tao W-Q. Numerical study of effective thermal conductivities of plain woven composites by unit cells of different sizes. International Journal of Heat and Mass Transfer. 2015,91:829-840.

[16] Gou J-J, Zhang H, Dai Y-J, Li S, Tao W-Q. Numerical prediction of effective thermal 
conductivities of 3D four-directional braided composites. Composite Structures. 2015,125:499-508.

[17] Lee S-E, Yoo J-S, Kang J-H, Kim C-G. Prediction of the thermal conductivities of four-axial non-woven composites. Composite Structures. 2009,89(2):262-269.

[18] Kumar S, Kumar A, Shukla A, Devi GR, Gupta AK. Thermal-diffusivity measurement of 3D-stitched C-SiC composites. Journal of the European Ceramic Society. 2009,29(3):489-495.

[19] Dong K, Gu B, Sun B. Comparisons of thermal conductive behaviors of epoxy resin in unidirectional composite materials. Journal of Thermal Analysis and Calorimetry. 2016,124(2):775-89.

[20] Suplicz A, Hargitai H, Kovacs JG. Methodology development for through-plane thermal conductivity prediction of composites. International Journal of Thermal Sciences. 2016,100:54-59.

[21] Ma F, Zhang P, Xia ZZ, Li M. How to enhance the effective thermal conductivity of composite material based on optimization method? Energy. 2015,87:400-411.

[22] Jin L, Hu H, Sun B, Gu B. Three-point bending fatigue behavior of 3D angle-interlock woven composite. Journal of Composite Materials. 2012,46(8):883-894.

[23] Xia Z, Zhang Y, Ellyin F. A unified periodical boundary conditions for representative volume elements of composites and applications. International Journal of Solids and Structures. 2003,40(8):1907-1921.

[24] Hibbit H, Karlsson B, Sorensen E. ABAQUS User Manual, Version 6.12. Simulia, Providence, RI. 2012 .

[25] Gustafsson SE. Transient plane source techniques for thermal conductivity and thermal diffusivity measurements of solid materials. Review of Scientific Instruments. 1991,62(3):797-804.

[26] He Y. Rapid thermal conductivity measurement with a hot disk sensor. Thermochimica Acta. 2005,436(1-2):122-129.

[27] Hasselman D, Donaldson K, Thomas J. Effective thermal conductivity of uniaxial composite with cylindrically orthotropic carbon fibers and interfacial thermal barrier. Journal of Composite Materials. 1993,27(6):637-644. 


\section{Table 1}

Specifications of 3DAWF.

\begin{tabular}{ccc}
\hline Parameters & Warp fiber tows & Weft fiber tows \\
\hline Linear density (tex) & 396 & 800 \\
Weave density (ends/cm) & 8 & 6.8 \\
Layers & 7 & 0.46 \\
Area density $\left(\mathrm{g} / \mathrm{cm}^{2}\right)$ & 6 \\
Fabric thickness $(\mathrm{mm})$ & & \\
\hline
\end{tabular}

Table 2

Basic parameters of fibers and the volume fraction of fibers in yarns.

\begin{tabular}{cccccc}
\hline Fiber's direction & Specification & $d(\mu \mathrm{m})$ & $S_{f}\left(\mathrm{~mm}^{2}\right)$ & $n_{f}$ & $\delta_{f}(\%)$ \\
\hline Warp & T300/6K & 7 & 0.32 & 6000 & 67.8 \\
Weft & T700/12K & 7 & 0.6476 & 12000 & 70.4 \\
\hline
\end{tabular}

Table 3

The volume fraction of fibers, resin matrix and voids in composites.

\begin{tabular}{ccccc}
\hline Method & Warp fibers $(\%)$ & Weft fibers $(\%)$ & Matrix (\%) & Voids (\%) \\
\hline Experimental & 21.08 & 24.86 & 52.86 & 1.2 \\
FEA & 21.96 & 25.19 & 51.65 & 1.2 \\
\hline
\end{tabular}

\section{Table 4}

Basic thermal properties of carbon fiber, epoxy resin and voids at room temperature.

\begin{tabular}{ccccc}
\hline \multirow{2}{*}{ Type } & Density $\left(\mathrm{g} \mathrm{cm}^{-3}\right)$ & \multicolumn{2}{c}{ Thermal conductivity $\left(\mathrm{W} \mathrm{m}^{-1} \mathrm{~K}^{-1}\right)$} & Specific heat $\left(\mathrm{J} \mathrm{kg}^{-1} \mathrm{~K}^{-1}\right)$ \\
\cline { 3 - 4 } WP (fiber) & 1.76 & $\mathrm{k}_{11}$ & $\mathrm{k}_{22}=\mathrm{k}_{33}$ & 755 \\
WF (fiber) & 1.8 & 9 & 1.178 & 750 \\
Epoxy resin & 1.13 & 10.145 & 1.256 & 1200 \\
Voids & 0.001293 & & 0.18 & \\
\hline
\end{tabular}




\section{Table 5}

Meshing scheme of multi-scale unit cell models.

\begin{tabular}{|c|c|c|c|c|}
\hline \multirow{2}{*}{\multicolumn{2}{|c|}{$\frac{\text { Scale }}{\text { Unit cell }}$}} & \multicolumn{2}{|c|}{ Micro-scale } & Meso-scale \\
\hline & & Fiber-matrix RVE & Matrix-voids RVE & Composite unit cell \\
\hline \multicolumn{5}{|c|}{$\begin{array}{l}\text { Schematic diagram } \\
\text { of meshing }\end{array}$} \\
\hline \multicolumn{2}{|c|}{ Size $(\mathrm{mm})$} & $1 \times 1.8 \times 0.5$ & $1 \times 1 \times 1$ & $8.8 \times 8.46 \times 6.26$ \\
\hline \multirow{7}{*}{$\begin{array}{l}\text { Number of } \\
\text { nodes }\end{array}$} & Total & 58707 & 29337 & 221993 \\
\hline & Edge $\mathrm{x}$ & $49,49,49,49$ & $50,50,50,50$ & $83,83,83,83$ \\
\hline & Edge y & $89,89,89,89$ & $50,50,50,50$ & $88,88,88,88$ \\
\hline & Edge z & $9,9,9,9$ & $9,9,9,9$ & $25,25,25,25$ \\
\hline & Face xy & 5057,5057 & 2463,2463 & 6565,6565 \\
\hline & Face yz & 810,810 & 450,450 & 2244,2244 \\
\hline & Face $x z$ & 441,441 & 450,450 & 2075,2075 \\
\hline \multirow{3}{*}{ Element } & Type & DC3D8 & DC3D8 & DC3D8 \\
\hline & Number & 51250 & 25590 & 307108 \\
\hline & Shape & Hexahedron & Hexahedron & Hexahedron \& tetrahedron \\
\hline \multicolumn{2}{|c|}{ Boundary conditions } & Full constraints & Full constraints & Partial constraints \\
\hline
\end{tabular}

\section{Table 6}

Comparison the thermal conductivities from different experimental methods.

\begin{tabular}{cccc}
\hline Method & TH direction & WA direction & WF direction \\
\hline DZDR-S & $0.9576 \pm 0.015$ & ---- & ---- \\
Self-designed apparatus & $0.983 \pm 0.032$ & $2.3763 \pm 0.057$ & $3.8245 \pm 0.084$ \\
\hline
\end{tabular}


Table 7

Thermal properties of matrix-voids RVE and fiber-matrix RVE (yarn model) at room temperature

\begin{tabular}{|c|c|c|c|c|c|}
\hline \multirow{2}{*}{ Type } & \multirow{2}{*}{$\begin{array}{c}\text { Voids } \\
\text { content }\end{array}$} & \multirow{2}{*}{ Density $\left(\mathrm{g} \mathrm{cm}^{-3}\right)$} & \multicolumn{2}{|c|}{ Thermal conductivity $\left(\mathrm{W} \mathrm{m}^{-1} \mathrm{~K}^{-1}\right)$} & \multirow{2}{*}{ Specific heat $\left(\mathrm{J} \mathrm{kg}^{-1} \mathrm{~K}^{-1}\right)$} \\
\hline & & & $\mathrm{k}_{11}$ & $\mathrm{k}_{22}=\mathrm{k}_{33}$ & \\
\hline \multirow{4}{*}{ Matrix-voids } & $1.2 \%$ & 1.102 & \multicolumn{2}{|c|}{0.1761} & 1190 \\
\hline & $5 \%$ & 1.074 & \multicolumn{2}{|c|}{0.1722} & 1200 \\
\hline & $10 \%$ & 1.017 & \multicolumn{2}{|c|}{0.1644} & 1200 \\
\hline & $1.2 \%$ & 1.557 & 6.162 & 0.642 & 854 \\
\hline \multirow[t]{2}{*}{ WP (yarn) } & $5 \%$ & 1.539 & 6.158 & 0.639 & 855 \\
\hline & $10 \%$ & 1.52 & 6.154 & 0.634 & 850 \\
\hline \multirow[t]{2}{*}{ With interface } & $1.2 \%$ & 1.557 & 6.16 & 0.641 & 854 \\
\hline & $1.2 \%$ & 1.602 & 7.2 & 0.685 & 840 \\
\hline \multirow[t]{2}{*}{ WF (yarn) } & $5 \%$ & 1.585 & 7.193 & 0.679 & 840 \\
\hline & $10 \%$ & 1.568 & 7.19 & 0.674 & 836 \\
\hline With interface & $1.2 \%$ & 1.602 & 7.198 & 0.68 & 840 \\
\hline
\end{tabular}

\section{Table 8}

Thermal conductivity of 2.5D AWC calculated by different methods and in different directions.

\begin{tabular}{cccc}
\hline Method & TH direction & WP direction & WF direction \\
\hline Experiment (1.2\% voids) & 0.983 & 2.3763 & 3.8245 \\
FEA (1.2\% voids) & 1.0235 & 2.5262 & 3.98 \\
FEA (5\% voids) & 0.97 & 2.1074 & 3.7176 \\
FEA (10\% voids) & 0.941 & 1.83 & 3.512 \\
FEA (1.2\% voids with interface) & 0.9978 & 2.394 & 3.8593 \\
\hline
\end{tabular}



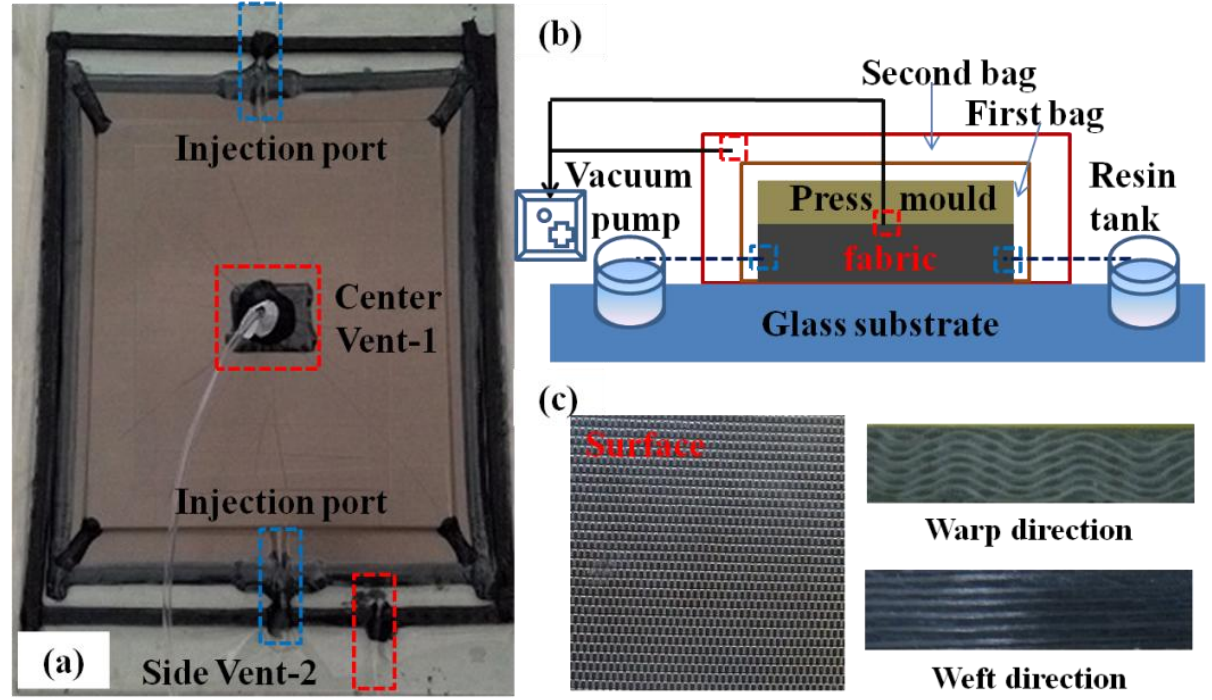

(c)

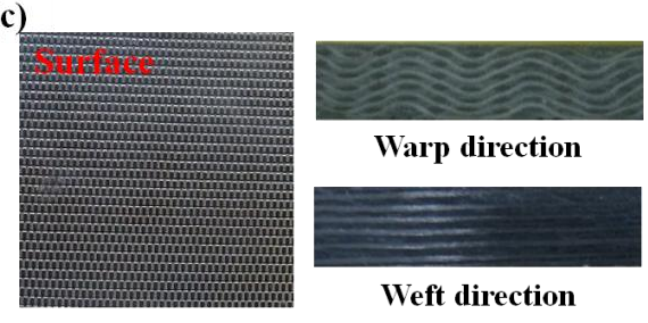

Figure.1. (a) Photograph of double vacuum insulation bags; (b) schematic diagram of VARTM; (c) photographs of composite coupons.

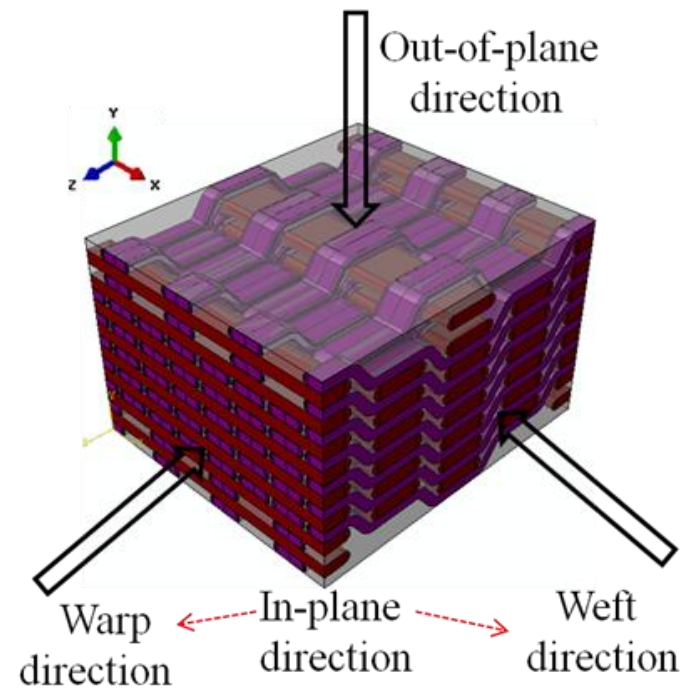

Figure.2. The definition of in-plane and out-of-plane directions. 


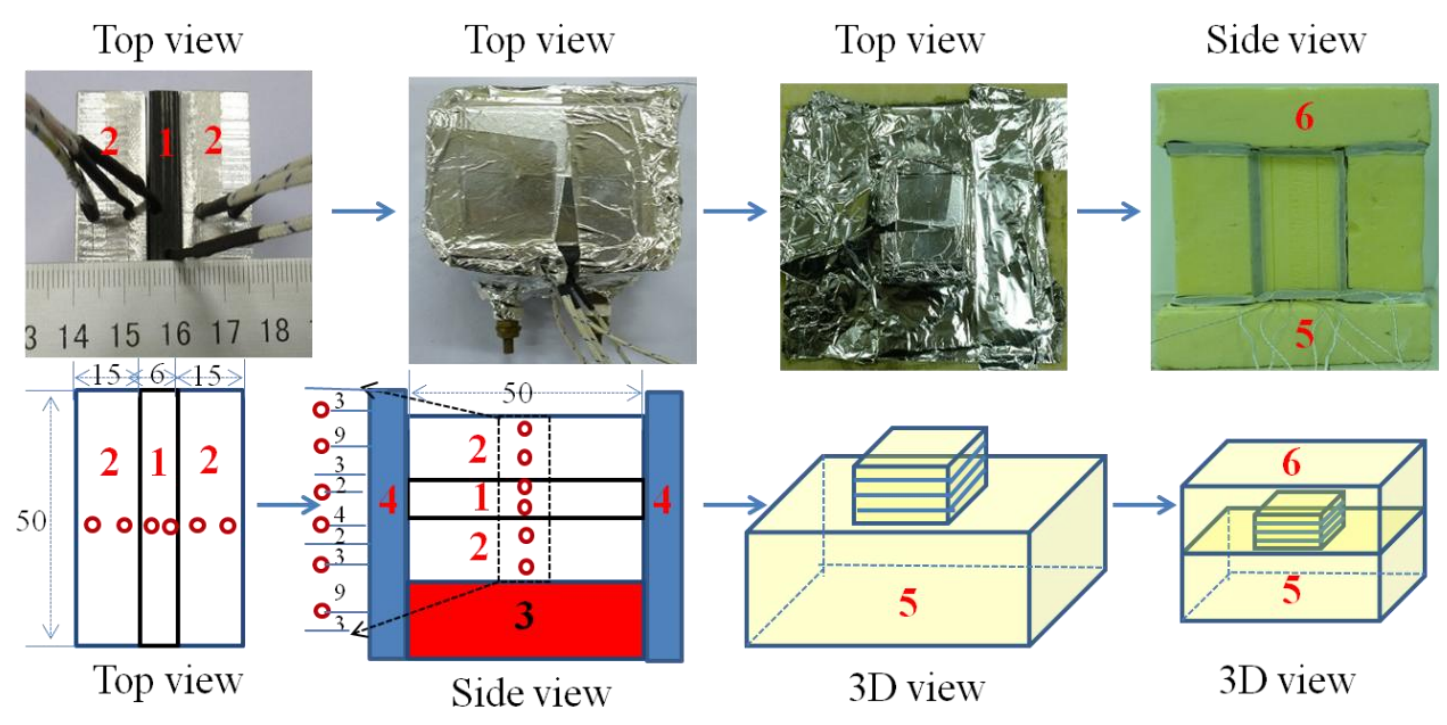

(a)

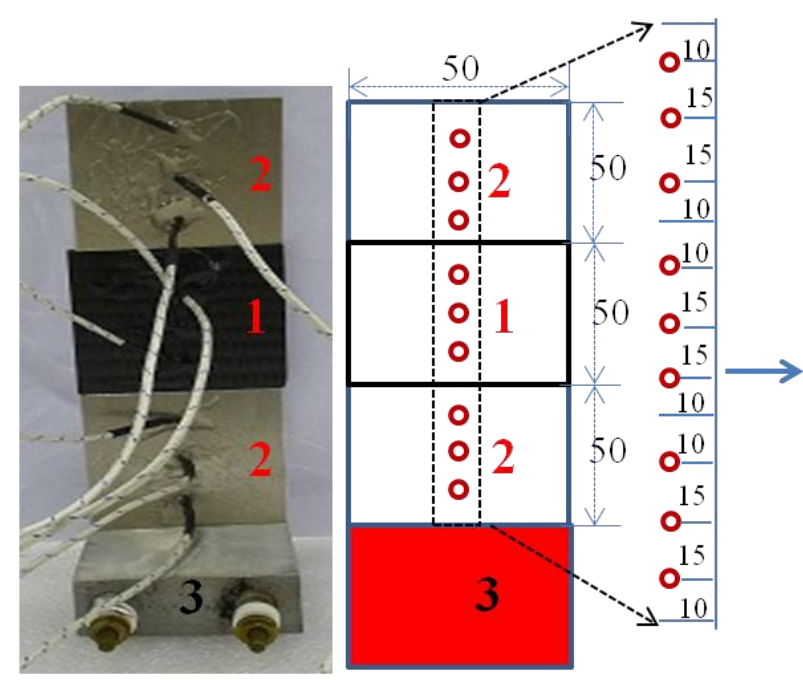

Frontal view

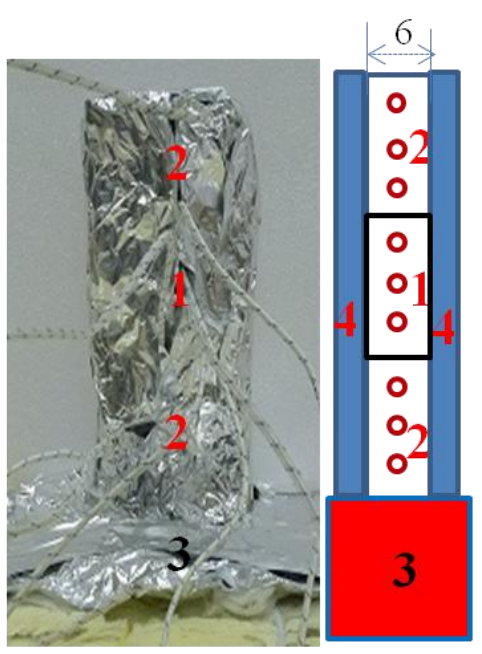

Frontal view Side view

(b)

Figure.3. Self-designed thermal conductive experimental apparatus for composite materials: (a) measurement along out-of-plane direction; (b) measurement along in-plane direction. (Notes: the

figures 1-6 refer to the composite coupon, the reference aluminum 5052 plate, the heating aluminum plate, alumina fiber insulating board, the lower foam board and upper foam cover board, respectively. The unit is $\mathrm{mm}$.) 


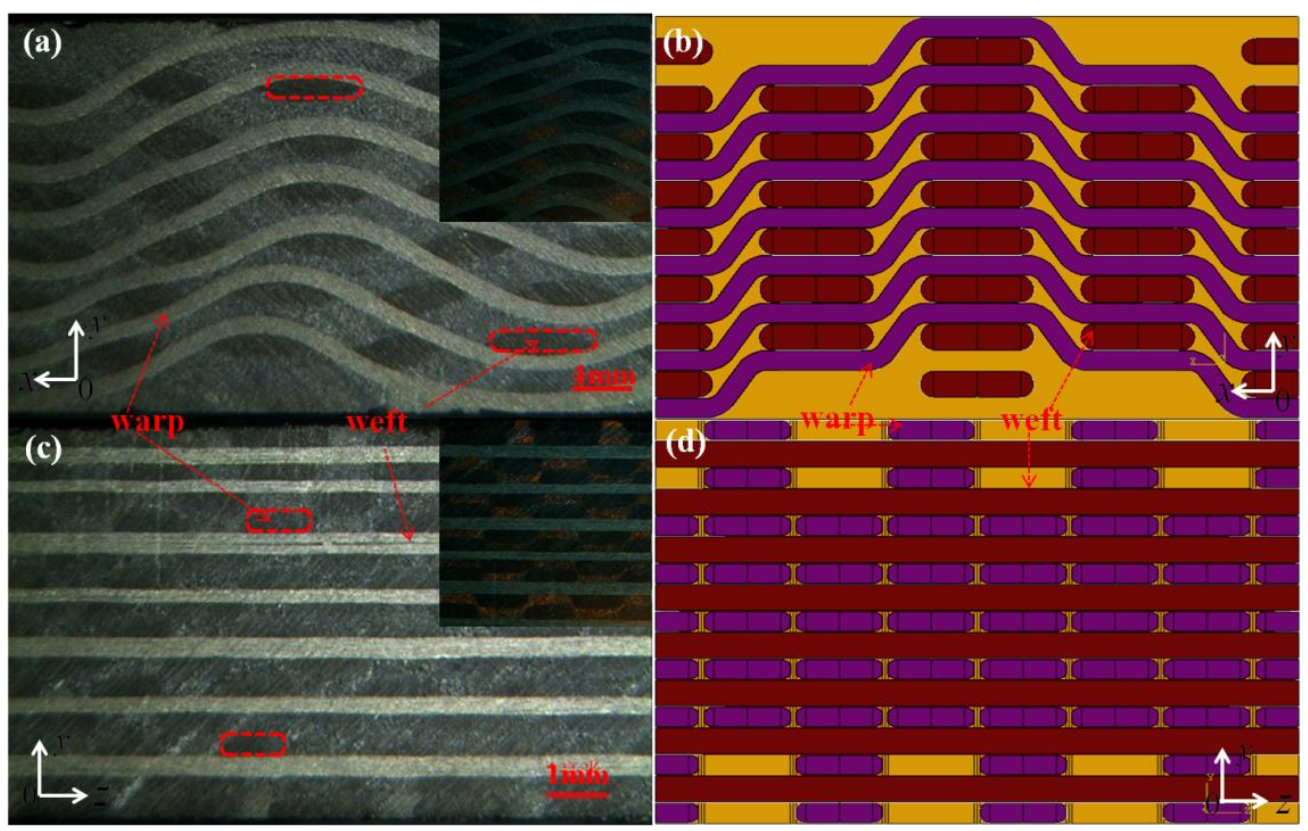

Figure.4. Geometrical microstructure of 2.5DAWC from optical microscopy: (a) cross-section photograph along warp direction; (b) cross-section schematic diagram along warp direction; (c) cross-section photograph along weft direction; (d) cross-section schematic diagram along weft direction.

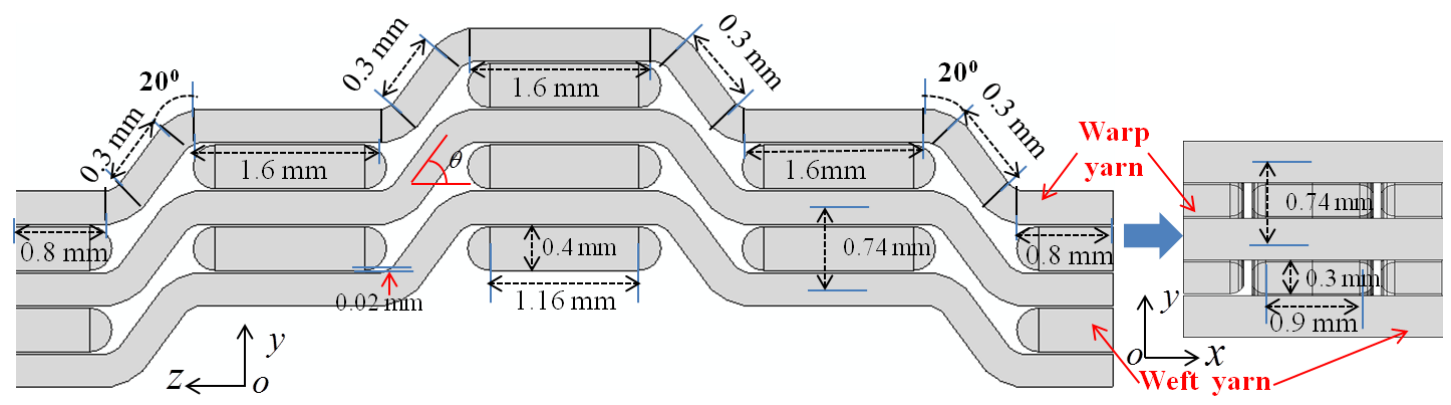

Figure.5. Illustration of the parameters in the 2.5DAWC.

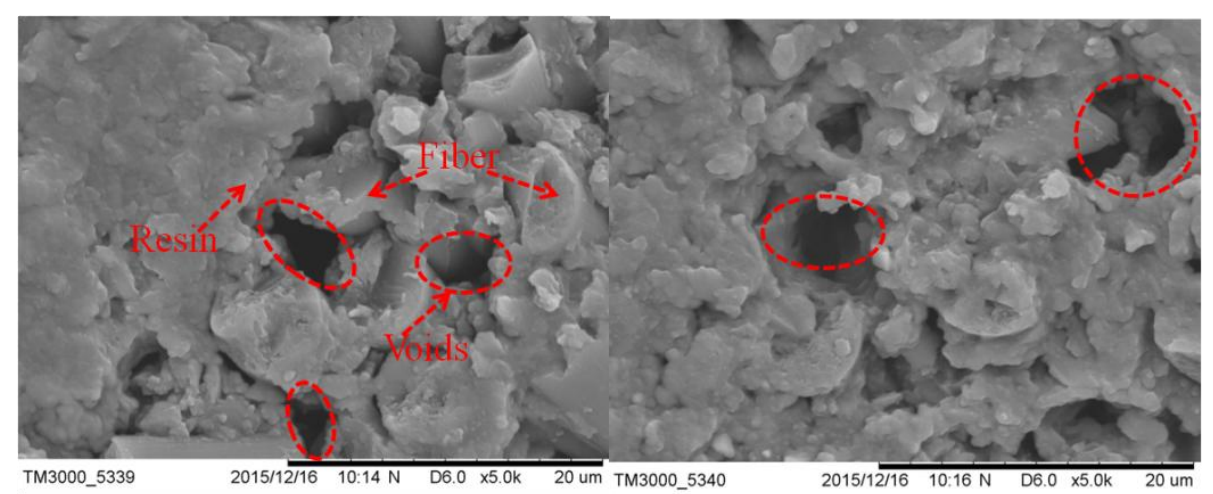

Figure.6. SEM photographs of voids in 2.5DAWC. 

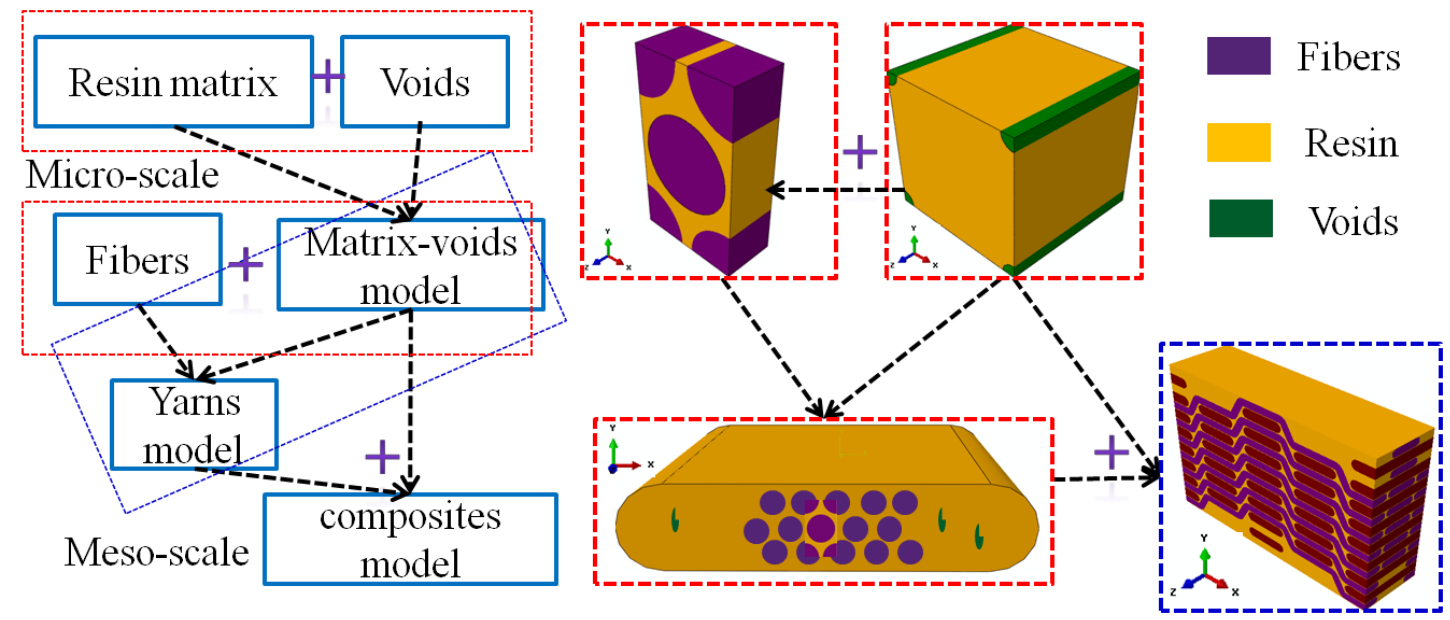

Figure.7. Multi-scale models of 2.5DAWC in FEA.

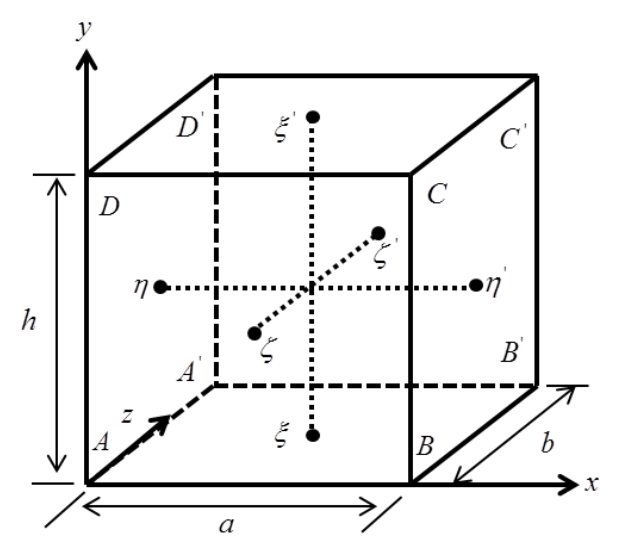

(a)

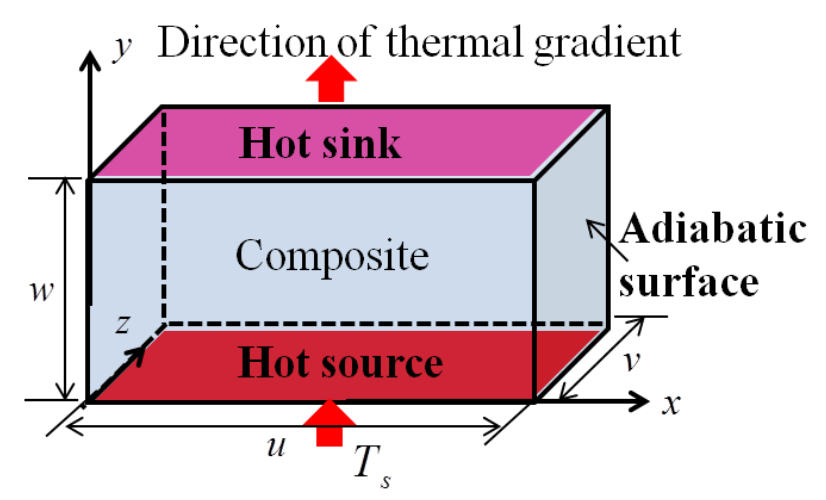

(b)

Figure.8. Boundary conditions used for FEA: (a) periodical boundary conditions; (b) general boundary condition used for simulation of out-of-plane direction. (Notes: $\xi, \eta$ and $\zeta$ refer to the nodes on the surfaces.)

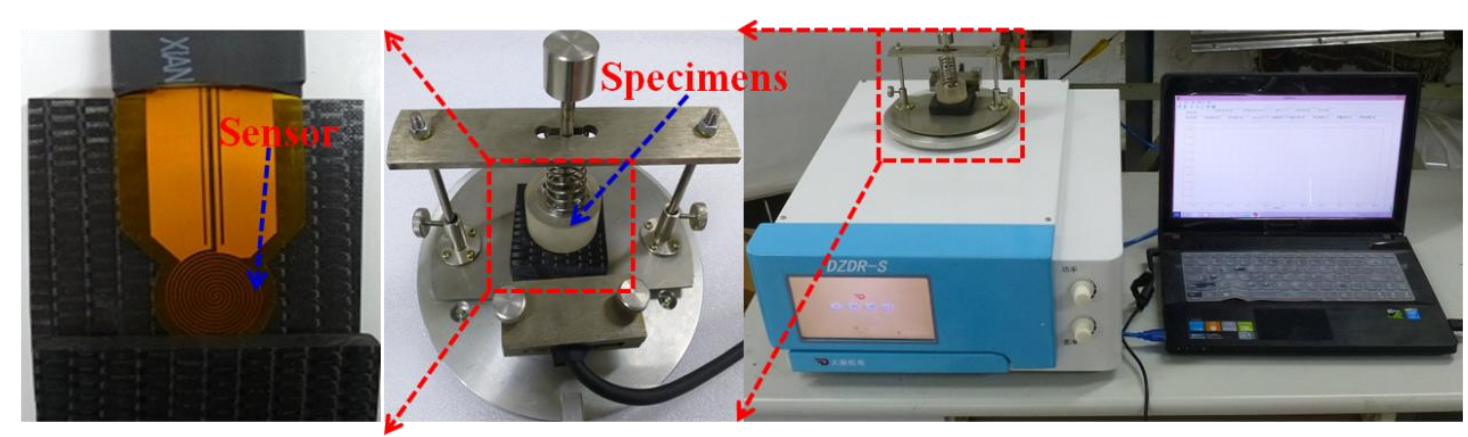

Figure.9. Supplementary experiment of measuring the thermal conductivity of 2.5DAWC. 


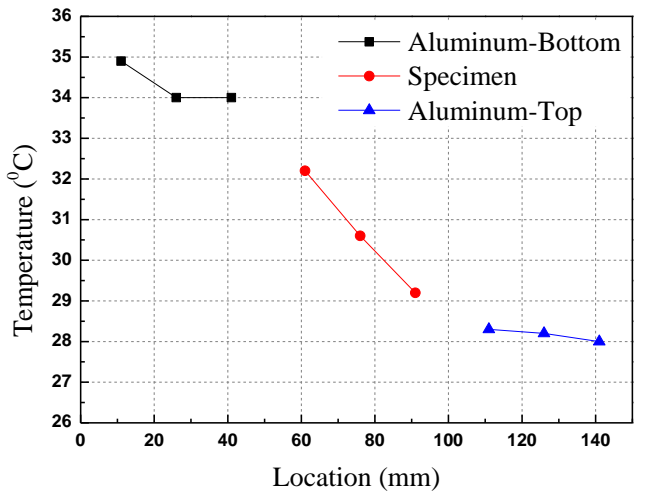

(a)

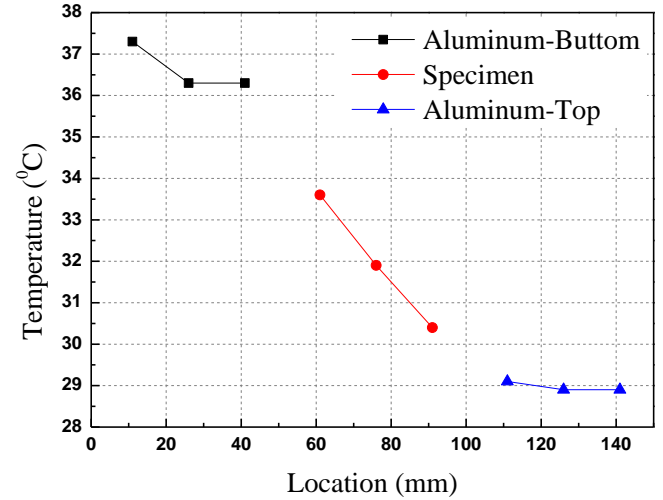

(b)

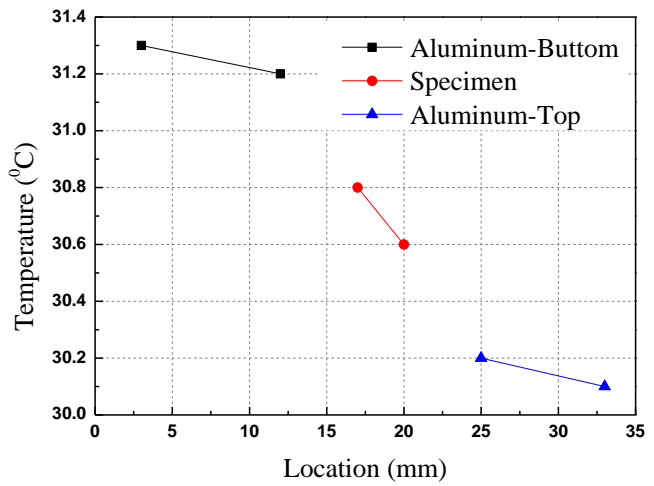

(c)

Figure.10. Average temperature distribution in the experimental measurement: (a) warp (WP)

direction; (b) weft (WF) direction; (c) out-of-plane (through-thickness) direction.

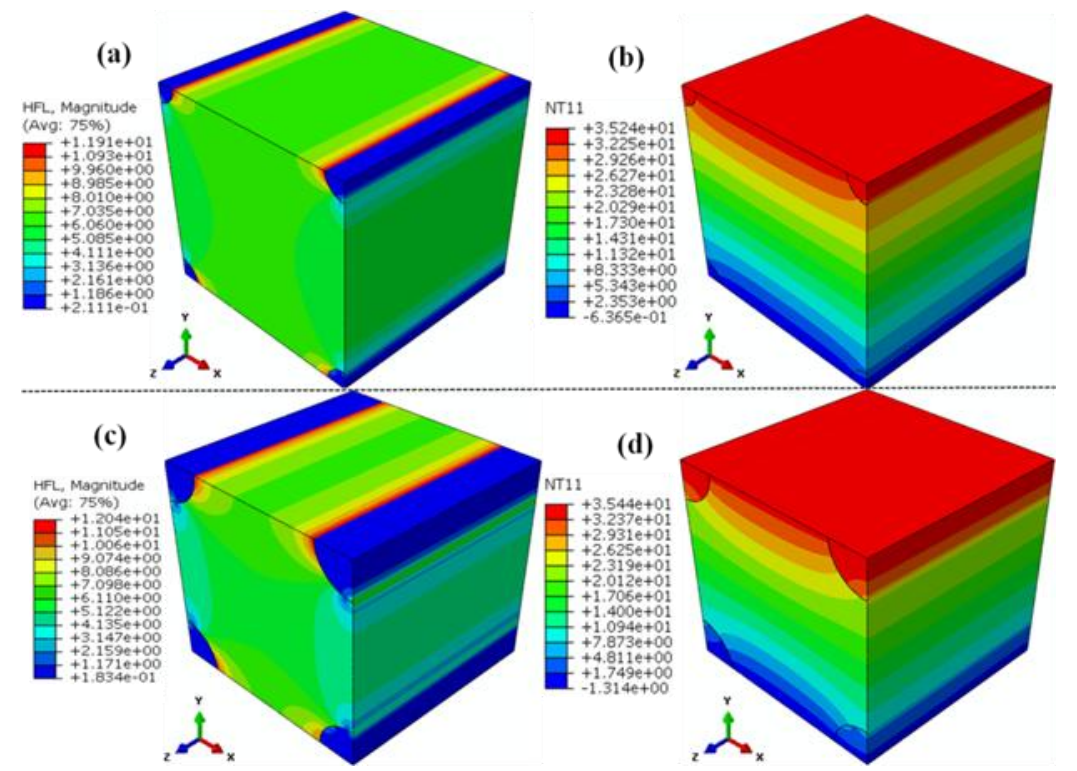

Figure.11. Heat flux (HF) and temperature (T) distributions of matrix-voids RVE: (a) HF of $1.2 \%$ voids; (b) T of $1.2 \%$ voids; (c) HF of $10 \%$ voids; (d) T of $10 \%$ voids.

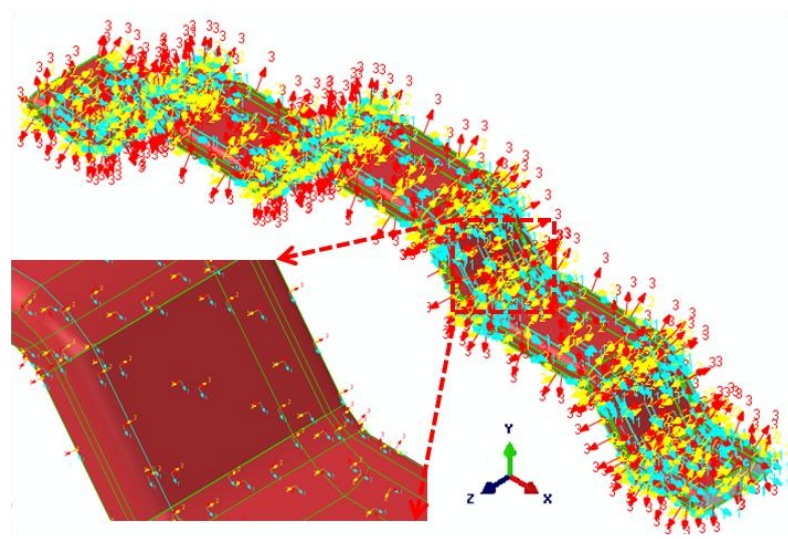

Fig.12. Orientation definition of one fiber tow treated as transversely isotropic UD composite. 


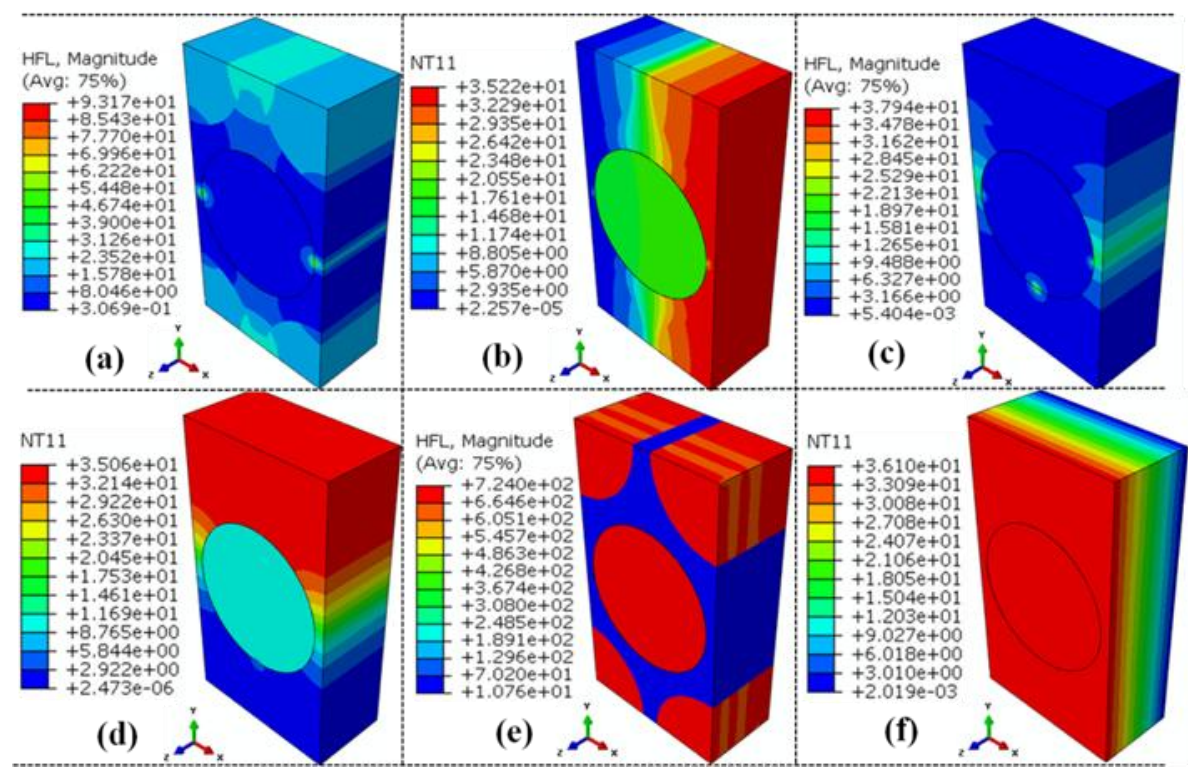

Figure.13. Heat flux (HF) and temperature (T) distributions of fiber-matrix RVE (yarn): (a) HF along 33 direction; (b) T along 33 direction; (c) HF along 22 direction; (d) $\mathrm{T}$ along 22 direction; (e) HF along 11 direction; (f) $\mathrm{T}$ along 11 direction.

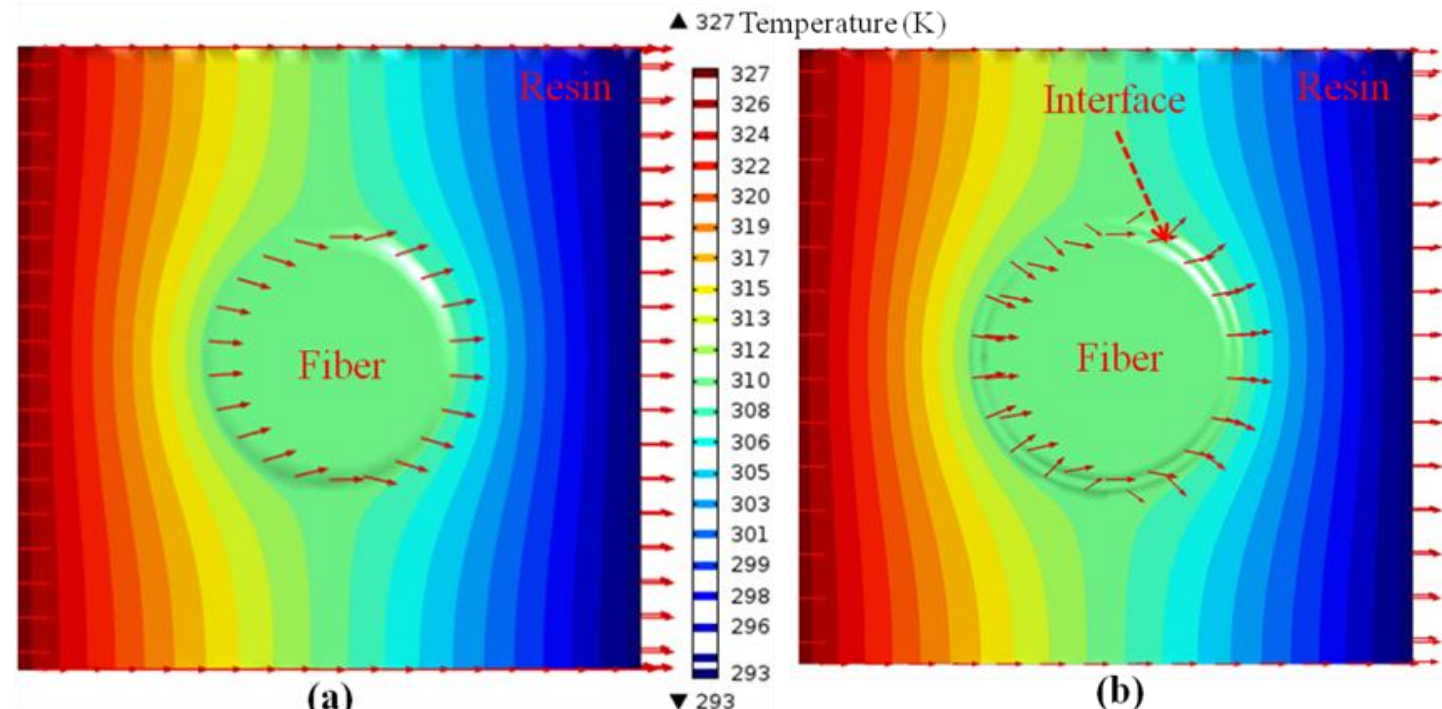

Figure.14. Isothermal contours and heat flux lines of fiber-matrix system under steady-state (stationary) condition: (a) without interface; (b) with interface. 


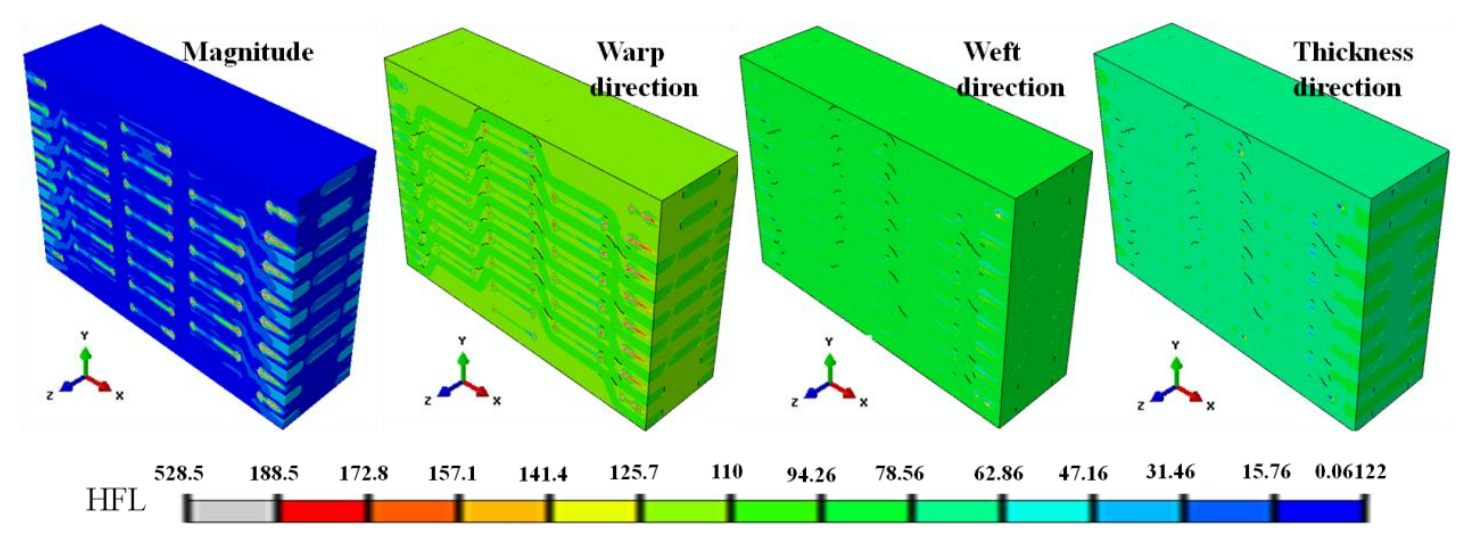

Figure.15. The distributions of heat flux in different directions by applying thermal gradient in warp direction.

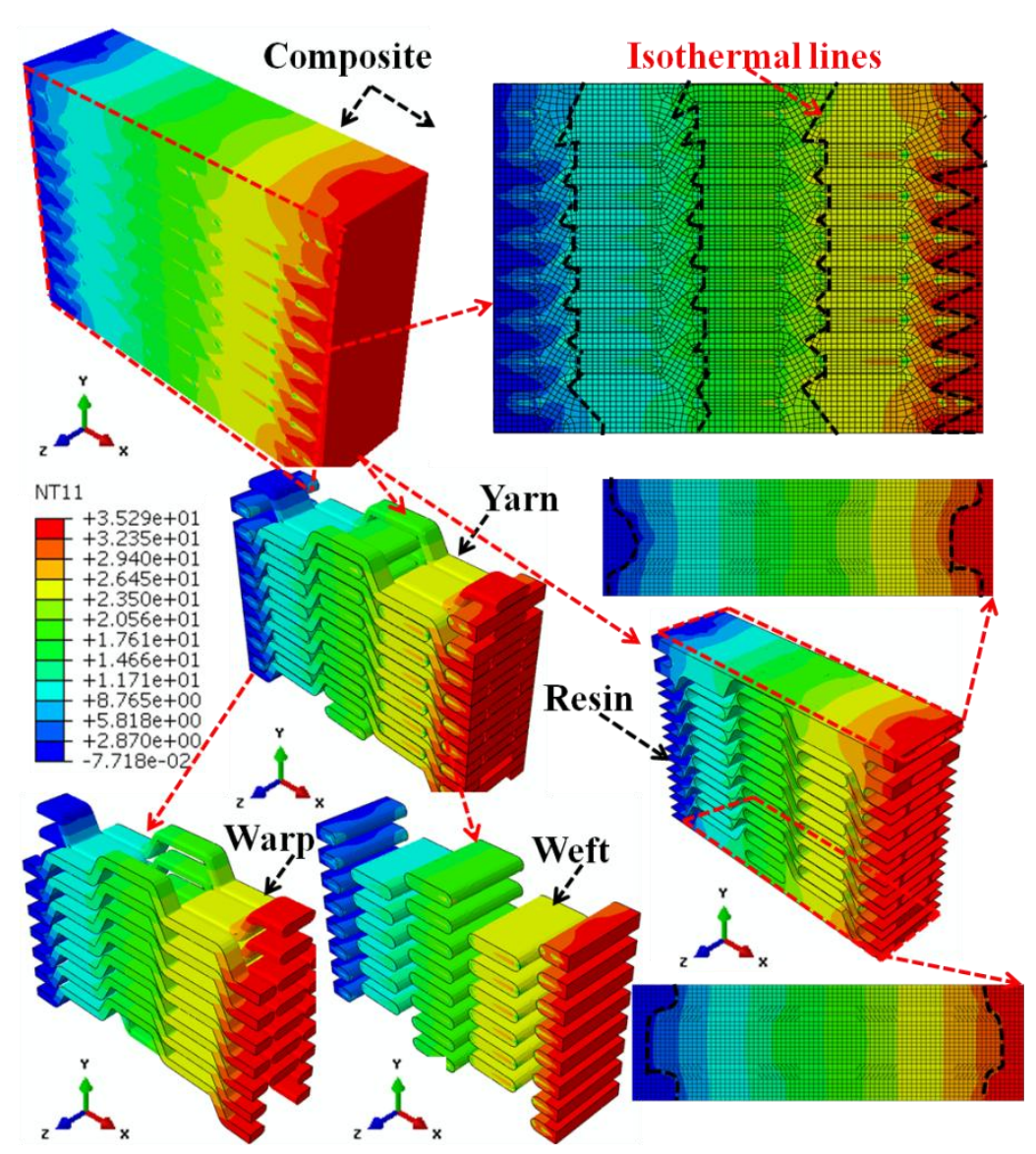

Figure.16. The distributions of temperatures by applying thermal gradient in warp direction. 


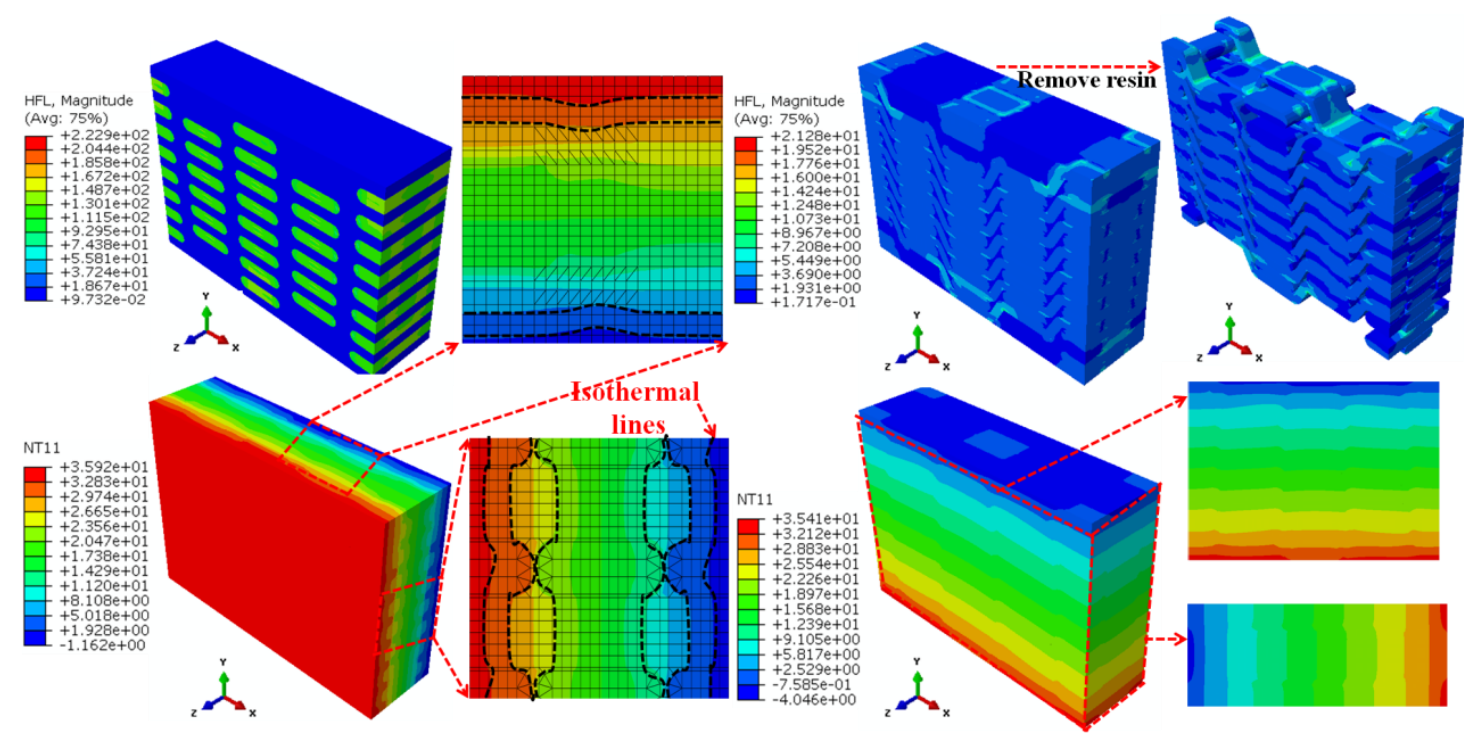

(a)

(b)

Figure.17. The distributions of heat flux and temperatures of meso-scale RVE: (a) applying thermal gradient in the weft direction; (b) applying thermal gradient in the thickness direction.

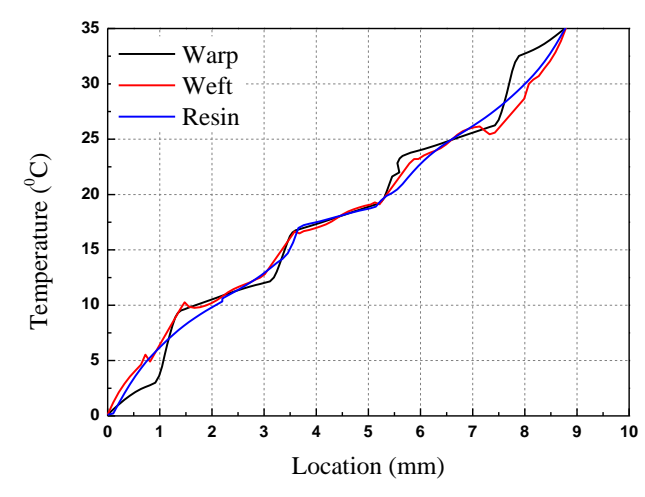

(a)

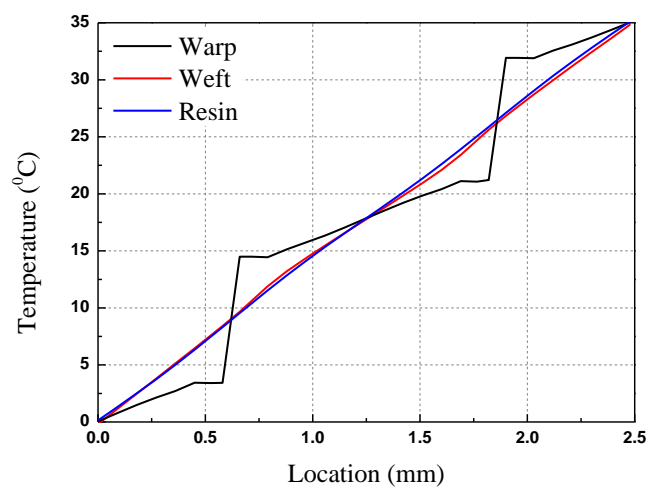

(b)

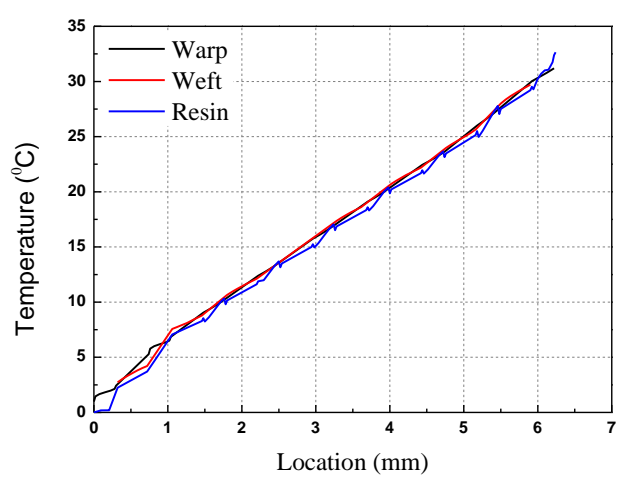

(c)

Figure.18. The average temperatures of meso-scale RVE along different directions: (a) along warp

(xx) direction; (b) along weft (zz) direction; (c) along through-thickness (yy) direction. 


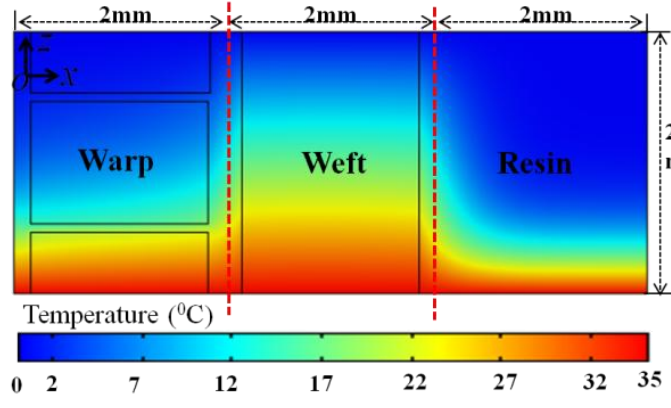

(a)

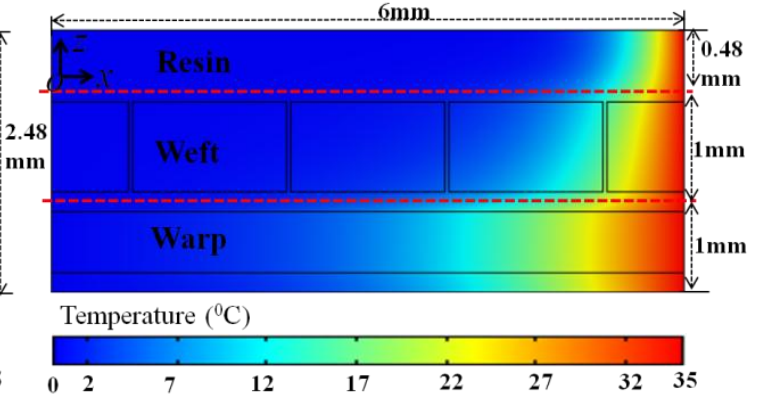

(b)

Figure.19. Transient (time dependent) thermal conductivity analyses along different directions: (a) along weft direction of composite; (b) along warp direction of composite. 